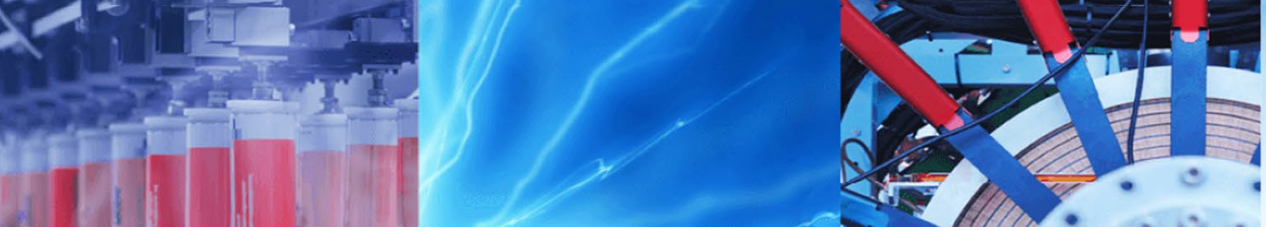

Review Paper

\title{
A review of the use of simple time-domain NMR/MRI for material-science
}

\author{
Time-domain benchtop and mobile NMR/MRI for liquid and solid material science, and nano- \\ to micro-pore-size measurement
}

\section{J. Beau W. Webber ${ }^{1}$ (I)}

Received: 5 May 2021 / Accepted: 31 August 2021

Published online: 27 September 2021

(C) The Author(s) 2021 OPEN

\begin{abstract}
The intention of this discussion is as a simple introduction for general-non-nuclear magnetic resonance (NMR)-specialist-materials scientists, to make them aware as to how some of the materials science measurements that they need to make might possibly be addressed by simple physical measurements using low-cost time-domain NMR apparatus. The intention is to include a minimum of complex NMR detail, while enabling general material-scientists to see that simple easily understood time-domain NMR might be of use to them. That is how I have tried to structure this discussion. It seems to me be generally forgotten how much of materials science is actually physics, as opposed to chemistry, and the extent to which simple time-domain NMR may be used to make measurements of the physical properties of materials. There frequently seems to be an assumption that if NMR is mentioned that it is chemical analysis methods that are under discussion, or possibly magnetic resonance imaging (MRI). These are both extremely powerful techniques, but to forget about the physics that often governs the properties of the sample can be a significant mistake. Key material science properties are often described in different fields using the terms mobility/dynamics/stiffness/viscosity/rigidity of the sample. These properties are usually dependent on atomic and molecular motion in the sample. We will discuss a method, time-domain NMR, that appears often to be ignored, to obtain quantitative or comparative information on these properties. The intention of this paper is not to probe the material properties of some interesting system, but to discuss in as clear a manner as possible a particular technique, "low-field time-domain NMR", to bring this technique and its advantages to the attention of other material scientists. Thus we discuss time-domain NMR and MRI, as methods of measuring the physical properties of liquid and solid materials. Time-domain NMR is also a good technique for measuring pore-size distributions from the nano-meter to microns, using a technique known as NMR cryoporometry (NMRC). Standard MRI protocols may be combined with NMRC, so that spatial resolution of pore dimensions may also be obtained. Low-field time-domain NMR is, at its fundamentals, a very approachable and easily comparative technique, where the material properties may often be extracted from the time-domain data much more simply than from say high-field high-resolution spectral data. In addition, low-field time-domain NMR apparatus is typically a factor of 10 to 100 times cheaper than high-field high-resolution solid-state NMR systems.
\end{abstract}

$\triangle$ J. Beau W. Webber, Dr.BeauWebber@gmail.com, http://www.Lab-Tools.com, https://nmrspectrometer.lab-tools.com/ | ${ }^{1}$ Lab-Tools Ltd. (Nano-Science), Marlowe Innovation Centre, Marlowe Way, Ramsgate CT12 6FA, UK. 
Keywords Material-science -Time-domain - Time domain NMR · Low-field - Low field NMR · NMR relaxation . NMR cryoporometry · FPGA · Field programmable gate array · Benchtop · NMR mouse · Digital RF · Variable temperature $\cdot \mathrm{V}-\mathrm{T} \cdot$ Pore-size $\cdot$ Process monitoring

\section{Introduction: Key NMR concepts}

\subsection{NMR on the benchtop and in the field}

We will discuss time-domain nuclear magnetic resonance (NMR) and magnetic resonance imaging (MRI), as methods of measuring the physical properties of liquid and solid materials. In particular, we consider why analyse the data in the time-domain measurements, as opposed to transforming the captured time-domain data to the frequencydomain and analysing it there.

The discussion will be very practical, giving many examples. Two tables of instrumental details on a number of common benchtop NMR spectrometers are given in Sect. 2. The examples have mainly been prepared using a particularly compact example, the lab-tools Mk3 timedomain NMR spectrometer [22, 24], see Fig. 1 and Sect. 2, unless otherwise stated. The 2D and 3D MRI Figs. 27, 28,29 , needed a wide-bore NMR time-domain imaging system. Some of the other benchtop NMR spectrometers have additional features that are useful for specific measurement needs.

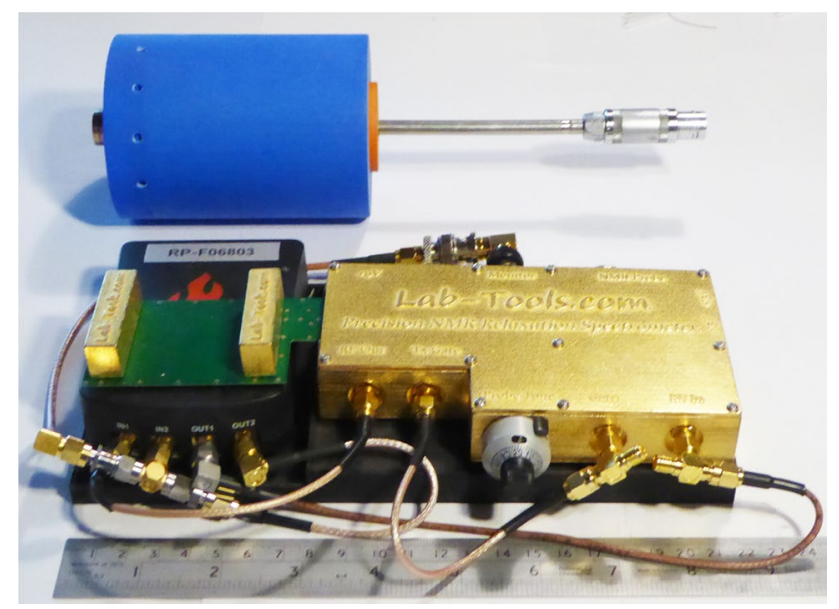

Fig. 1 A highly compact lab-tools Mk3 time-domain NMR spectrometer and light-weight mandhalas NMR magnet, both designed especially for low-field material science NMR relaxation and NMR cryoporometry pore size measurements, in the lab and in the field

\subsection{Time-domain NMR for material science}

Time-Domain NMR is excellent for quantified monitoring of physical change, particularly as a function of some changing parameter such as time or sample temperature.

Firstly, the key point about using time-domain NMR is that it provides a direct method to determining the physical motion of the atoms and molecules in the sample.

This leads to information on sample properties that in different fields may variously be described as the mobility/ dynamics/stiffness/viscosity/rigidity of the sample, particularly of solid hydrocarbons, rubbers and other polymers. This information may be analysed quantitatively, but for many purposes, such as process monitoring and control, comparative measurements are sufficient.

\subsection{Why does time-domain NMR provide a direct method to determining the physical motion in the sample?}

Some nuclei (such as hydrogen-i.e. in say water or a polymer) have a magnetic moment; their behaviour in a magnetic field is governed by quantum mechanics, but as we are considering the average motion of a very large number of spins, we may actually use a classical model :

Such a nucleus will precess (nutate) in a magnetic field $\left(B_{0}\right)$, like a spinning top does in the earth's gravitational field;

The $\mathrm{B}_{0}$ magnetic field at a given nucleus is modified by the magnetic field of adjacent nuclei, changing the rate at which the first nucleus precesses in the magnetic field.

If everything is rigid, this extra field has a long time to act, and so overall, the different nuclei in the sample rapidly get out of step with one another, and the net rotating magnetic field that we measure decays rapidly, perhaps in $10 \mu \mathrm{s}$.

As motion increasingly sets in, perhaps at higher temperatures, these field offsets start changing, and start averaging out over an NMR rotational period:

- "Motional Narrowing" sets in, resulting in a slower decay. We will later discuss specific examples.

\subsection{NMR relaxation times: the mechanisms by which decays take place in NMR and MRI measurements}

When an NMR measurement is made on a sample that has been placed in a strong steady $B_{0}$ magnetic field, a strong radio frequency (RF) pulse may be applied that perturbs the nuclear magnetism from its equilibrium position along (against) the main $B_{0}$ field. This 


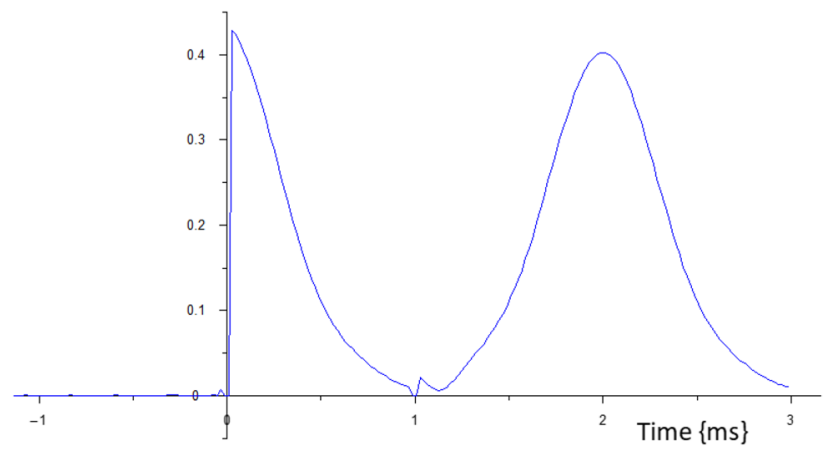

Fig. 2 A single-shot time-domain NMR trace, with free-induction decay (FID) and echo, in a $114 \mathrm{mg}$ hexadecane liquid sample in a standard $5 \mathrm{~mm}$ diameter NMR sample tube

magnetisation vector then rotates around the $B_{0}$ field at the Lamour angular frequency $\omega_{0}$ in a similar manner to a spinning top in the earth's gravitational field. This perturbed magnetisation of the nuclei creates a measurable signal that evolves with time. [1]. For a benchtop NMR spectrometer the NMR frequency $F$ is typically 2 to $100 \mathrm{MHz}$.

The angular frequency of this signal in a magnetic field $B$ is given by $\omega=|\gamma| B$ where the gyromagnetic ratio $\gamma_{p}$, for protons in water, is around $2.675222005(63) \times 10^{8}$ rads . $\mathrm{S}^{-1} \cdot \mathrm{T}^{-1}$.

In conventional frequency $\mathrm{F}$, this is given by $\left(\gamma_{p} / 2 \pi\right) \mathrm{B}$ which may be approximated as $42.58 \ldots \mathrm{MHz} \cdot \mathrm{T}^{-1}$.

One mechanism for the decay of this measured signal is a "spin-spin" interaction due to interaction between the spins of the nuclei-this causes a loss of transverse magnetisation, that then decays in a time known as the $T_{2}$ decay time.

Another mechanism for the decay of the measured signal is a "spin-lattice" interaction-this is in effect the equilibration (relaxation) of the nuclear magnetisation with the thermal-noise bath of the "lattice", back to its original equilibrium state, in a time known as the $\mathbf{T}_{\mathbf{1}}$ decay time.

$\mathrm{T}_{1}$ relaxation is caused by magnetic fluctuations ("spectral densities"), usually caused by thermal fluctuations, at 1 $x$ and $2 x$ the Lamour frequency $\omega_{0}: T_{2}$ is similar, but is also sensitive to static magnetic fields.

There are another two important decay times:

The $\mathbf{T}_{2}{ }^{*}$ decay time, which is a (recoverable) loss of the measured magnetisation signal, due to different spins being in different magnetic fields, and thus all getting increasingly out of step with one another as the system evolves. This may be due to a non-uniform $B_{0}$ field, or perhaps due to deliberately applied magnetic gradient fields, as in diffusional or MRI measurements. Further R.F. pulses may be applied to temporarily recover the measured magnetisation signal.

The $\mathbf{T}_{1} \boldsymbol{\rho}$ decay time, which is measured using long pulses of RF to "spin-lock" the nuclear magnetisation, following an initial $90^{\circ}$ pulse. This in effect measures the $T_{1}$ decay time in a magnetic field of the strength of the $B_{1} R F$ field, rather than the static $B_{0}$ field; thus it probes fluctuations at $\omega_{1}$.

Measuring at different $B_{0}$ and $B_{1}$ magnetic field strengths can provide vital additional information on the atomic and molecular motions. A particular advantage of using $\mathrm{T}_{1} \rho$ at lower pulse powers is that it probes the slower molecular motions.

To demonstrate an example as to what is typically measured, as a result of the above decay processes, here is a single-shot time-domain NMR trace, from an $114 \mathrm{mg}$ hexadecane liquid sample, using the above spectrometer, Fig. 2 :

Here we see a key advantage of working in the timedomain :

At zero time the first $\left(90^{\circ}\right)$ R.F pulse is applied, to tip the magnetisation from along the main $B_{0}$ magnetic field into the transverse $X-Y$ plane, where it precesses.

The measured (blue) signal trace at zero time has an amplitude that is directly proportional to the mass of the sample in the probe; this gives an extremely robust method, once the system gain is calibrated, of measuring components of this mass as probed by different NMR experiments.

The signal then evolves with time-the free-inductiondecay (FID). Here we are in a typical low-field low-cost permanent magnet, and the visible decay in the signal amplitude is determined by $\mathrm{T}_{2}{ }^{*}$ as the precessing nuclei in the different $B_{0}$ fields get out of step with one another.

After a delay of $1 \mathrm{~ms}$, a second RF pulse is applied, a $180^{\circ}$ pulse which flips the pancake of dephased spins over. The spins that were precessing faster and had gained in phase are now at an equal negative phase, and visa-versa. So after an equal length of time they will re-phase to produce an echo. The new amplitude will now have decayed from the amplitude at time zero, due to $\mathrm{T}_{2}$ processes.

This behaviour is shown diagrammatically in Fig. 3 .

A chain of $180^{\circ}$ may also be applied - the Carr-PurcellMeiboom-GIll sequence (CPMG). This is particularly useful for measuring $\mathrm{T}_{2}$ when it is longer than $\mathrm{T}_{2}{ }^{*}$. Figure 4 shows an example of an automated fit for water in sol-gel silica pores.

Time-domain NMR can thus be used to study these material science dynamical properties both in the bulk and in nano-meter and upward sized pores. 


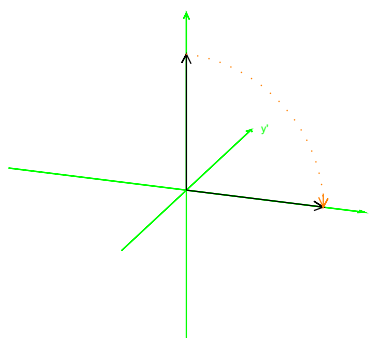

(a) Precession due to $90^{\circ} y^{\prime}$ R.F. pulse.

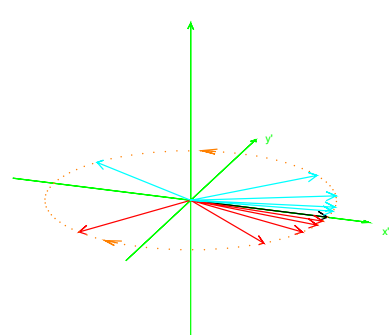

(b) Dephasing decay of FID due to field variations.

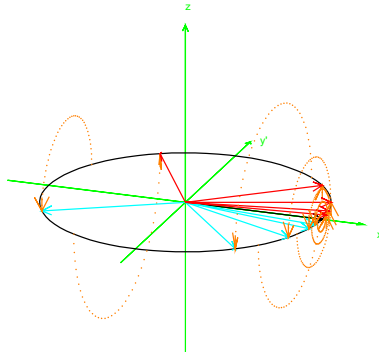

(c) Precession due to $180^{\circ}{ }_{x^{\prime}}$ R.F. pulse.

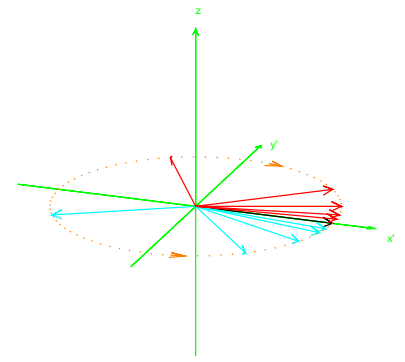

(d) Rephasing of spins to produce an echo.

Fig. 3 Generation of a spin echo in an inhomogeneous magnetic field using a $90^{\circ}{ }_{y^{\prime}}-\tau-180^{\circ}{ }_{x^{\prime}}-\tau$-echo sequence, where the blue vectors are in a higher field than the red ones, and thus precess faster

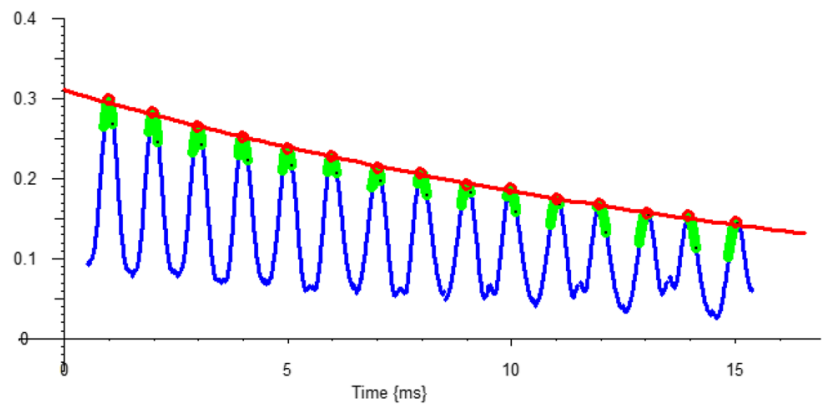

Fig. 4 An example of a chain of NMR Pulses in a Carr-Purcell-Meiboom-Gill sequence (CPMG) pulse sequence, for water in sol-gel silica pores. Each echo peak has been polynomial fitted, the polynomial algebraically solved to find the peak (red dots), and an exponential fit made to the peaks, giving the reduced $T_{2}$ of the water due to surface relaxation in the pores

\subsection{Using NMR to study the properties of structured or porous materials, and materials in these pores}

There are various methods for studying structured and or porous materials by time-domain NMR and MRI, which can "see inside" these structured materials and pores to measure their internal structure and properties :
A frequently used technique is to imbibe a liquid (or gas) into a porous structure, and then image the liquid, or study the changed properties of the liquid. A key point is frequently that when a nucleus in the liquid impinges on a pore wall then its magnetization signal (that we are measuring) is lost or scrambled, causing a faster NMR decay time on average in the pore. For many specimens, such as biological ones, foodstuffs or unfired clays, the necessary water is already inside the sample.

There are a number of key methods that are frequently used:

$$
\begin{aligned}
& \text { NMR relaxation (NMRR) } \\
& \text { NMR diffusion and percolation, } \\
& \text { NMR imaging (MRI) } \\
& \text { NMR relaxometry, } \\
& \text { NMR cryoporometry (NMRC) }
\end{aligned}
$$

\subsubsection{NMR relaxation (NMRR)}

For many structured samples, the NMR relaxation properties of the contained liquids are defined by which part of the structure that they reside within-highly important biological examples include cancerous vs non-cancerous cells, and nerve pathways. In rocks recovered from underground reservoirs, it is frequently found that there are very short decay bituminous fractions on the pore walls, but more mobile oily components in the body of the pore [23], see Sect. 2.7. Field-cycling relaxometry using variable field magnets is a powerful method of probing motions at different rates, as is $T_{1} \rho$, see Sect. 2.6.

\subsubsection{NMR diffusion and percolation}

These are very powerful methods of studying porous structures-Magnetic field gradients (both static and time varying) and NMR pulses are applied to a liquid in the pores during the NMR measurement-this can measure movement of the atoms of the liquid through the pores. A key strength of diffusional structure determination, vs imaging, is that for diffusional measurements the signalto-noise in the measured structure can be obtained as an average over many similar structures, not just one [17].

\subsubsection{NMR imaging (MRI)}

Again, magnetic field gradients (both static and time varying), often extremely long and complex sequences in 1,2 or 3 spatial dimensions, with long trains of NMR pulses, are applied to the object being studied, to produce images, 
that can have amazing detail, using Fourier and related Transforms. Large libraries of NMR pulse sequences are available. Combining MRI and NMRR protocols to produce functional images and 3D data sets has proved to be hugely informative. Image scales from whole-humanbody down to microns are possible, although the smaller scales suffer from signal-to-noise problems.

\subsubsection{NMR relaxometry}

This makes use of the faster NMR relaxation processes that, for a liquid in the pore, take place at the pore wall, as the diffusing nuclei impinge on the pore wall and coherence is lost [2]. This technique works well for some materials, particularly larger rock pores, such as sand-stones, providing broad pore-size distributions. The surface relaxivity needs to be known, to calculate actual pore dimensions, and in many substances, such as say shales or carbonates, this is not yet known in sufficient detail. Some research has shown that the surface relaxivities can be different even in nominally similar laboratory porous silicas prepared by different sources [9].

\subsubsection{NMR cryoporometry (NMRC)}

NMRC is a technique where the protocol is that one again adds a liquid to the pores, but this time freezes the liquid, and then uses NMRR as a robust method to measure the liquid melting point, which is inversely proportional to pore diameter. We have extended the NMRC technique for measuring distributions of pore sizes over 3 orders of magnitude in pore dimensions. We will discuss this technique further in Sect. 4. The NMRC protocol can also be combined with the MRI protocol, to produce $1 D, 2 D$ or $3 D$ resolved porosity [13-15], see Sect. 4.3.

\section{Example low-field time-domain NMR spectrometers}

There are quite a number of bench-top spectrometers produced by different companies. We have contacted many, and we discuss a set of example low-field time-domain NMR spectrometers, where we have received replies. See the table in Fig. 5 for smaller spectrometers, mostly benchtop and portable spectrometers, while the table in Fig. 6 is for larger spectrometers, mostly floor-standing, which in general have a wider range of options and capabilities.

We discuss in more detail an example highly compact low-field time-domain NMR spectrometer, the Lab-Tools Mk3, see Fig. 1 and the last entry in table 5, suitable for use on the laboratory bench, or in the field, to study solid or liquid samples. The cost of such a spectrometer, complete with low-field NMR magnet, is typically one to two orders of magnitude below that of a typical high-resolution solidstate NMR spectrometer with super-conducting magnet. Thus one can make low-field time-domain NMR material science measurements simply and at low cost.

In this particular device, the RF is processed digitally in the credit-card sized Field Programmable Gate Array (FPGA) module on the left, which also has a dual-core ARM Linux computer on the same FPGA chip. This digital RF

\begin{tabular}{|c|c|c|c|c|c|c|c|c|c|c|}
\hline Name & Maker & \begin{tabular}{|l|} 
NMR \\
Frequency \\
\end{tabular} & $\begin{array}{l}\text { Time- } \\
\text { Domain }\end{array}$ & $\begin{array}{l}\text { Frequency- } \\
\text { Domain }\end{array}$ & $\begin{array}{l}\text { Rigid } \\
\text { Solids }\end{array}$ & $\begin{array}{l}\text { Solid } \\
\text { Polymers }\end{array}$ & Liquids & T1Rho & Special Features & $\begin{array}{l}\text { Size / Weight } \\
\text { (Spectrometer-Magnet) }\end{array}$ \\
\hline Minispec & $\begin{array}{l}\text { Bruker } \\
\text { www.bruker.com }\end{array}$ & $\begin{array}{l}7.5 \mathrm{MHz} \\
20 \mathrm{MHz} \\
40 \mathrm{MHz} \\
60 \mathrm{MHz}\end{array}$ & Yes & No & Yes & Yes & Yes & Option & $\begin{array}{l}\text { Proton or Fluorine } \\
\text { Optional V-T } \\
\text { Optional PFG }\end{array}$ & Table Top \\
\hline MQC+ & $\begin{array}{l}\text { Oxford Instruments } \\
\text { nmr.oxinst.com }\end{array}$ & $\begin{array}{l}5 \mathrm{MHz} \\
23 \mathrm{MHz}\end{array}$ & Yes & No & Yes & Yes & Yes & No & $\begin{array}{l}5 \mathrm{MHz}: 60,50,40 \mathrm{~mm} \text { Proton probes } \\
\text { 23MHz: } 26,18^{*}, 10^{*}, 5 \mathrm{~mm} \text { Proton; } 26 \\
\mathrm{~mm} \text { Fluorine probes } \\
{ }^{*} \text { Liquid V-T options }-5 \text { to } 70^{\circ} \mathrm{C}\end{array}$ & Table top \\
\hline Spin Track & $\begin{array}{l}\text { Resonance Systems } \\
\text { www.nmr-design.com }\end{array}$ & $\begin{array}{l}\text { Spectrometer } \\
1 \mathrm{kHz} \text { to } 80 \\
\mathrm{MHz} ; \\
\text { Standards } \\
\text { Magnets: } \\
14 \mathrm{MHz} \text {, } \\
18 \mathrm{MHz}, \\
20 \mathrm{MHz} \\
58 \mathrm{MHz}\end{array}$ & Yes & With FFT & Yes & Yes & Yes & Yes & $\begin{array}{l}\text { Options: } \\
\text { Scripting of Sequences; } \\
\text { V-T from }-60 \mathrm{C} \text { to } 200 \mathrm{C} ; \\
\text { Autosampler; } \\
\text { PFG } 1 \text { or } 3 \text { Axis, Flow Probe; } \\
\text { Double Frequency Probe; Surface } \\
\text { Probe; } \\
\text { Nuclei: }{ }^{1 \mathrm{H},}{ }^{2 \mathrm{H}},{ }^{19} \mathrm{~F},{ }^{31} \mathrm{P} .{ }^{23} \mathrm{Na},{ }^{27} \mathrm{Al},{ }^{57} \mathrm{Fe} \text {, } \\
\text { }{ }^{29} \mathrm{Si} ; \\
\text { Earth Field; } \\
\text { loT Automation }\end{array}$ & \\
\hline $\begin{array}{l}\text { Turbospec } \\
\text { e400 }\end{array}$ & $\begin{array}{l}\text { Turbospec LLC } \\
\text { http://turbospecllc.com/ }\end{array}$ & Broadband & Yes & No & No & No & Yes & No & Quantitative Elemental Analysis & $8.3 \mathrm{~kg}$ \\
\hline Mk3 & $\begin{array}{l}\text { Lab-Tools } \\
\text { https://nmrspectrometer.lab- } \\
\text { tools.com/ }\end{array}$ & $\begin{array}{l}\text { Broadband } \\
5 \text { to } 30 \mathrm{MHz} \\
\text { or wider }\end{array}$ & Yes & No & Yes & Yes & Yes & Yes & $\begin{array}{l}\text { Proton or Fluorine } \\
\text { Optional V-T - } 70 \mathrm{C} \text { to } 120 \mathrm{C} \\
\text { NMR Cryoporomety pore sizes. } \\
\text { Large bore magnets for V-T probes, etc. }\end{array}$ & $\begin{array}{l}\text { Spectrometer : 1kg } \\
\text { Magnets : } \\
<1 \mathrm{~kg} 12 \mathrm{MHz} \text {, } \\
1 \mathrm{~kg} 21 \mathrm{MHz} \text {, } \\
60 \mathrm{kG} 23 \mathrm{MHz} \text {. } \\
\text { All } 35 \mathrm{~mm} \text { gap. }\end{array}$ \\
\hline
\end{tabular}

Fig. 5 Benchtop/portable time-domain NMR spectrometers 


\begin{tabular}{|c|c|c|c|c|c|c|c|c|c|c|}
\hline Name & Maker & \begin{tabular}{|l|} 
NMR \\
Frequency
\end{tabular} & $\begin{array}{l}\text { Time- } \\
\text { Domain }\end{array}$ & $\begin{array}{l}\text { Frequency- } \\
\text { Domain }\end{array}$ & \begin{tabular}{|l|} 
Rigid \\
Solids
\end{tabular} & $\begin{array}{l}\text { Solid } \\
\text { Polymers }\end{array}$ & Liquids & T1Rho & Special Features & $\begin{array}{l}\text { Size / Weight } \\
\text { (Spectrometer- } \\
\text { Magnet) }\end{array}$ \\
\hline MQR & $\begin{array}{l}\text { Oxford Instruments } \\
\text { nmr.oxinst.com }\end{array}$ & $20 \mathrm{MHz}$ & Yes & No & Yes & Yes & Yes & Yes & $\begin{array}{l}\text { 26, } 18^{*}, 10^{*} \mathrm{~mm} \text { Proton; } 26,10 \mathrm{~mm} \text { Fluorine } \\
\text { probes } \\
\text { Options: bipolar PFG; 2D expts, e.g. T1-T2, T2- } \\
\text { Diffusion, T2-store-T2, spatially resolved T2, etc; } \\
\text { 1, } 2 \text { and 3-D MRI, } \\
\text { *Liquid V-T - } 10 \text { to } 80^{\circ} \mathrm{C} \text { and Gas V-T } \\
-30 \text { to } 150^{\circ} \mathrm{C} \text { options }\end{array}$ & $\begin{array}{l}\text { Magnet unit - } \\
\text { Table top, } \\
\text { Electronics } \\
\text { console - Floor } \\
\text { standing }\end{array}$ \\
\hline GeoSpec 2 & $\begin{array}{l}\text { Oxford Instruments } \\
\text { nmr.oxinst.com }\end{array}$ & $2 \mathrm{MHz}$ & Yes & No & Yes & Yes & Yes & No & $\begin{array}{l}2 \mathrm{MHz}: 75,53,43,33,29 \mathrm{~mm} \text { standard proton } \\
\text { probes, } 1 \text { inch overburden cell (proton probe, } \\
\text { max pressure } 5 \mathrm{kpsi} \text {, temperature control up to } \\
100^{\circ} \mathrm{C} \text { ), } 1.5 \text { inch overburden probe (proton probe, } \\
\text { max pressure } 2.5 \mathrm{kpsi} \text {, temperature control up to } \\
100^{\circ} \mathrm{C} \text { ), } 50 \% \text { duty cycle RF-amplifier } \\
\text { Options: bipolar PFG, } 2 \text { D experiments, e.g. T1- } \\
\text { T2, T2-Diffusion, T2-store-T2, spatially resolved } \\
\text { T2, etc; } 1,2 \text { and } 3-D \text { MRl; } \\
\text { Gas-VT (option): from room temperature to } 60^{\circ} \mathrm{C}\end{array}$ & $\begin{array}{l}\text { Magnet unit - } \\
\text { Table top, } \\
\text { Electronics } \\
\text { console - Floor } \\
\text { standing }\end{array}$ \\
\hline GeoSpec 12 & $\begin{array}{l}\text { Oxford Instruments } \\
\text { nmr.oxinst.com }\end{array}$ & $12 \mathrm{MHz}$ & Yes & Yes & Yes & Yes & Yes & No & $\begin{array}{l}12 \mathrm{MHz}: 53,43,33,29 \mathrm{~mm} \text { standard proton } \\
\text { probes, } 1 \text { inch overburden cell (proton probe, } \\
\text { max pressure } 5 \mathrm{kpsi} \text {, temperature control up to } \\
100^{\circ} \mathrm{C} \text { ), } 1.5 \text { inch overburden probe (proton probe, } \\
\text { max pressure } 2.5 \mathrm{kpsi} \text {, temperature control up to } \\
100^{\circ} \mathrm{C} \text { ), } 50 \% \text { duty cycle RF-amplifier } \\
\text { Options: bipolar PFG, } 2 \mathrm{D} \text { experiments, e.g. T1- } \\
\mathrm{T} 2 \text {, T2-Diffusion, T2-store-T2, spatially resolved } \\
\mathrm{T} 2 \text {, etc; } 1,2 \text { and 3-D MRI }\end{array}$ & $\begin{array}{l}\text { Magnet unit - } \\
\text { Table top, } \\
\text { Electronics } \\
\text { console - Floor } \\
\text { standing }\end{array}$ \\
\hline Mag Station & $\begin{array}{l}\text { LexMar Global Inc. } \\
\text { https:/llexmarglobal.com/ }\end{array}$ & $20 \mathrm{MHz}$ & Yes & No & Yes & Yes & No & No & Auto sampler & Floor Standing \\
\hline MagModule & $\begin{array}{l}\text { LexMar Global Inc. } \\
\text { https://lexmarglobal.com/ }\end{array}$ & $20 \mathrm{MHz}$ & Yes & No & Yes & Yes & No & No & Online unit with custom sampler & Floor Standing \\
\hline $\begin{array}{l}\text { SMARtracer } \\
\text { - FFC NMR } \\
\text { relaxometer }\end{array}$ & $\begin{array}{l}\text { Stelar s.r.l } \\
\text { www.steler.it }\end{array}$ & $\begin{array}{l}0.005 \text { to } 0.25 \\
\text { Tesla }\end{array}$ & Yes & $\begin{array}{l}\text { System RF } \\
1-150 \mathrm{MHz}\end{array}$ & Yes & Yes & Yes & Yes & $\begin{array}{l}\text { T1-T2-2D T1/T2 } \\
\text {-field dependence of Magnetic Relaxation from } \\
0.005 \text { to } 0.25 \text { Tesla } \\
\text { Temperature range from }-140 \text { to }+140{ }^{\circ} \mathrm{C}\end{array}$ & $80 \mathrm{Kg}$ \\
\hline
\end{tabular}

Fig. 6 Larger/floor-standing time-domain NMR spectrometers

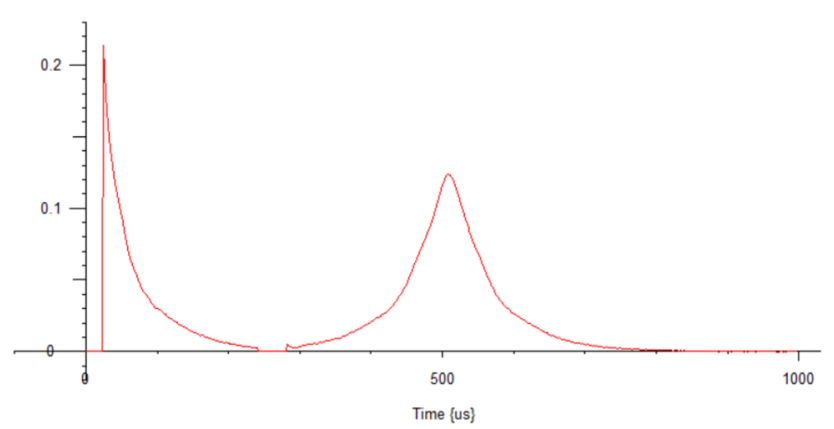

Fig. 7 An NMR FID and Echo from a small $5 \mathrm{~mm}$ OD water sample, measured in the Mandhalas magnet

processing helps give long-term stability, which greatly helps long duration process measurements, and helps give an extremely compact instrument.

\subsection{Compact NMR magnets}

Compact NMR magnets can be constructed using multiple magnet segments. Halbach arrays [7] are examples of such compact magnet arrays that can give highly homogeneous fields, suitable for chemical resolution. A lab-tools low homogeneity Mandhalas magnet [12] (less than $1 \mathrm{~kg}$ in weight) is shown in Fig. 1 that is constructed from 12 square cross-section magnet bars.

Figure 7 shows an NMR FID and Echo from a small $5 \mathrm{~mm}$ OD water sample, demonstrating the homogeneity with this Mandhalas magnet. This shows that even such a simple and compact magnet can be used to make useful time-domain NMR measurements. Such an NMR spectrometer and magnet can easily be used in the field, taking the spectrometer to the sample, rather than the sample to the spectrometer. There are also numerous examples of hand-held NMR magnets in the literature [5], that could be adapted. 


\subsection{Using a time-domain NMR spectrometer to measure mobility/dynamics/stiffness/ viscosity/rigidity}

As we have seen, the motion of atoms gives rise to a spectral-density of magnetic fluctuations that directly modify the various relaxation times as measured by NMR; in particular the $T_{1}$ Lattice decay, the $T_{2}$ spin-spin decay, and also $\mathrm{T}_{1 \rho}$ in the Rotating Frame, which gives fairly direct information on the slower molecular motions.

There are standard NMR pulse sequences to measure many of these relaxation times: $\mathbf{A}: \mathrm{FID}: \mathrm{T}_{2} \mathrm{~S}$ shorter than FID;

A-B, CPMG : $\mathrm{T}_{2}$ s longer than FID;

\section{A-A, B-A : $T_{1} s$;}

T1Rho: $: T_{1} \rho: T_{1}$ in the rotating-frame.

\subsection{Lab-tools T-D NMR spectrometer with peltier cooled variable-temperature NMR probe}

A very wide range of techniques have been used over the years to vary the temperature of NMR probes and samples, including using direct injection of liquid nitrogen droplets [11], bubbling highly dried air through liquid nitrogen and then warming it up, use of electrically evaporated liquid nitrogen gas, and commercial laboratory recirculating chillers.

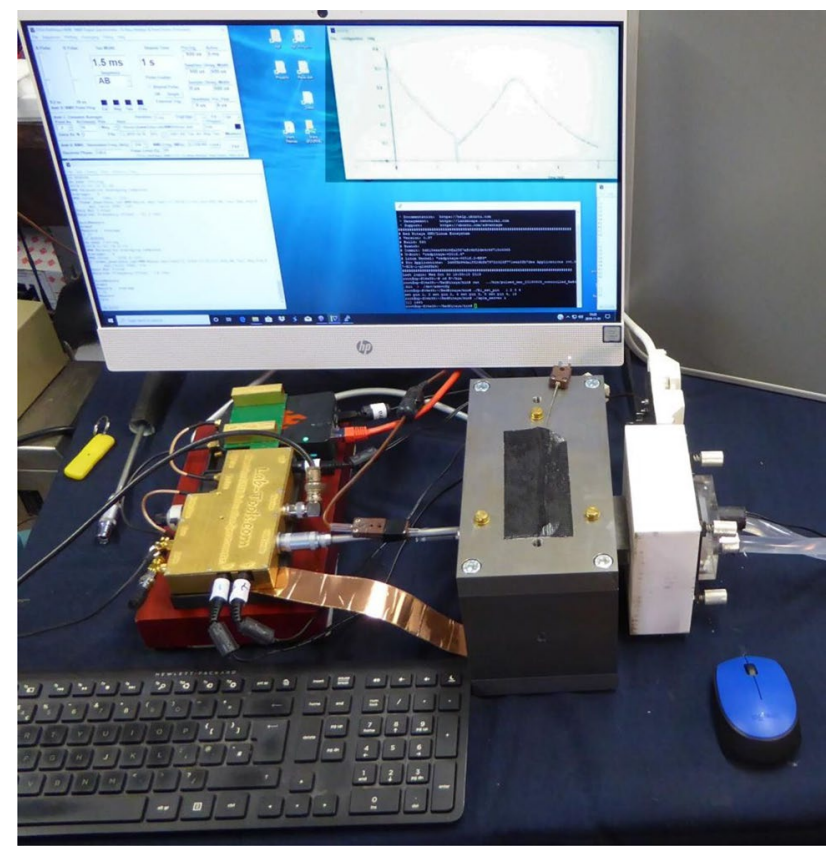

Fig. 8 The lab-tools NMR spectrometer is shown with variable-temperature NMR Probe, with the Peltier thermo-electric cooling elements in the white box. The room-temperature liquid waste heat removal circuit can be seen on the right
Our preferred technique now is to use Peltier thermoelectric cooling ( $-35 \mathrm{C}$ to $+80 \mathrm{C})$, aided to $-80 \mathrm{C}$ with millisecond bursts of $\mathrm{CO}_{2}$ gas. The Pelter cooling gives superb long-term precision control of temperature, which is essential for NMR Cryoporometric pore-size- distribution measurements from sub- $1 \mathrm{~nm}$ to over $1 \mu \mathrm{m}$ (needing $10 \mathrm{mk}$ precision near the probe liquid bulk melting point) [22].

\section{Gaussian Fitted Ice FIDs}
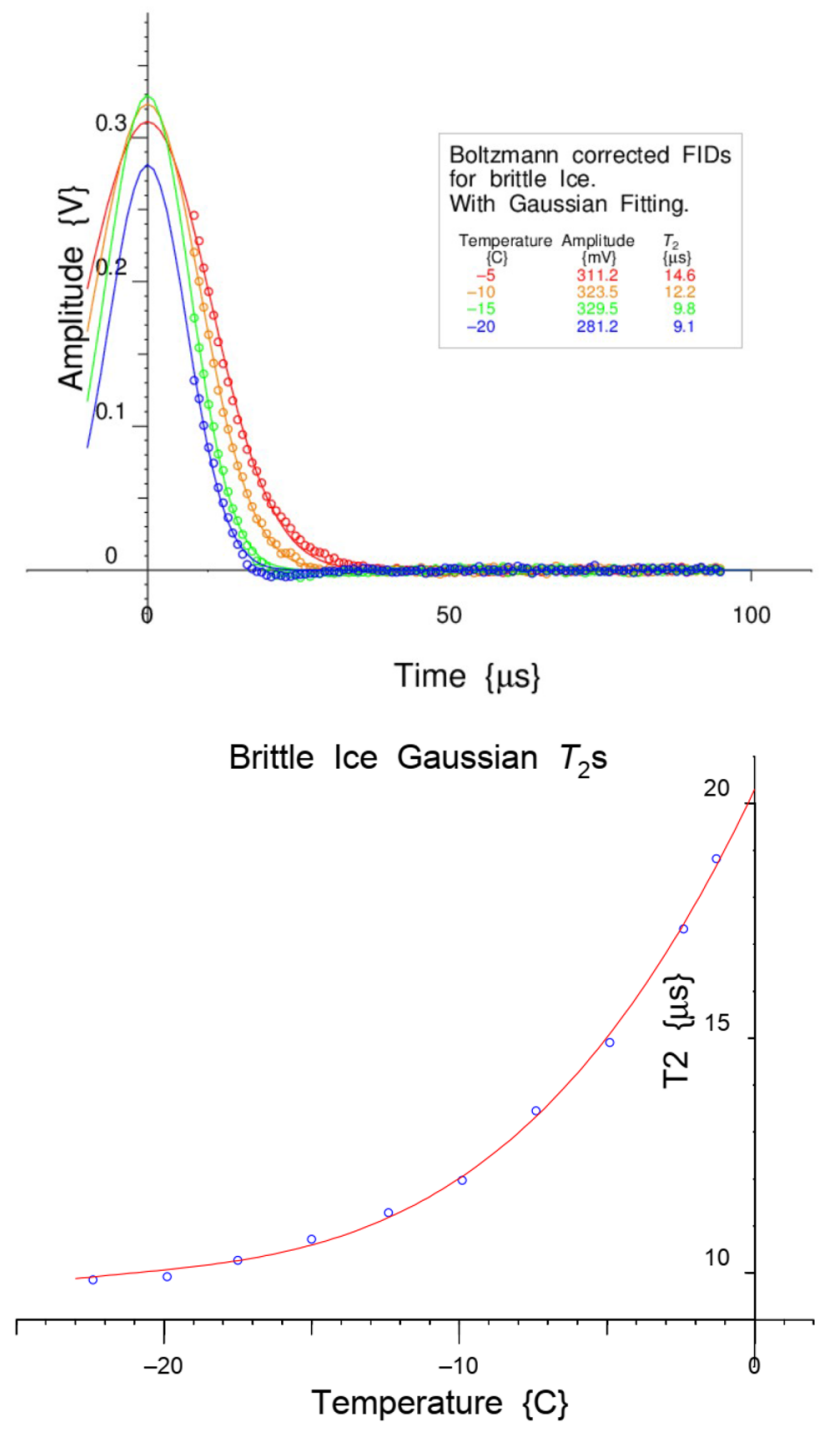

Fig. 9 A set of $T_{2}$ measurements using time-domain NMR which is sensitive to changes in the motion of the ice molecules in brittle ice, $-22 \mathrm{C}$ to melting 


\subsection{NMR Mk3 cryoporometer}

The NMR cryoporometer is controlled by a multi-tasking Apl array processing program, which also measures the temperatures and analyses the results.

The spectrometer communicates with the windows computer over the ethernet, using .net and TCP communications. The current multi-stage Peltier thermo-electrically cooled probe can function with room-temperature air cooling. Currently the waste heat is removed from the Peltier coolers using a water to air cooling circuit Fig. 8, although a recirculating laboratory chiller could be used. The advantage of using additional Peltier thermo-electrical cooling even when using a recirculating laboratory chiller is a lower achievable temperature and a far higher precision of temperature control.

\subsection{Motion of the molecules in Brittle Ice, studied using NMR $\mathrm{T}_{2}$ measurements}

Key material science properties are often described in different fields using the terms mobility/dynamics/stiffness/ viscosity/rigidity of the sample. These properties are usually dependent on atomic and molecular motion in the sample. We now look at the case of using Time-Domain NMR to observe the changes in the motion of brittle ice molecules as a function of temperature, Fig. 9. Even in such a rigid material the motion of the molecules may be measured. As is often the case with very hard and brittle materials, the
$T_{1 \rho}$ Measurements on Perspex

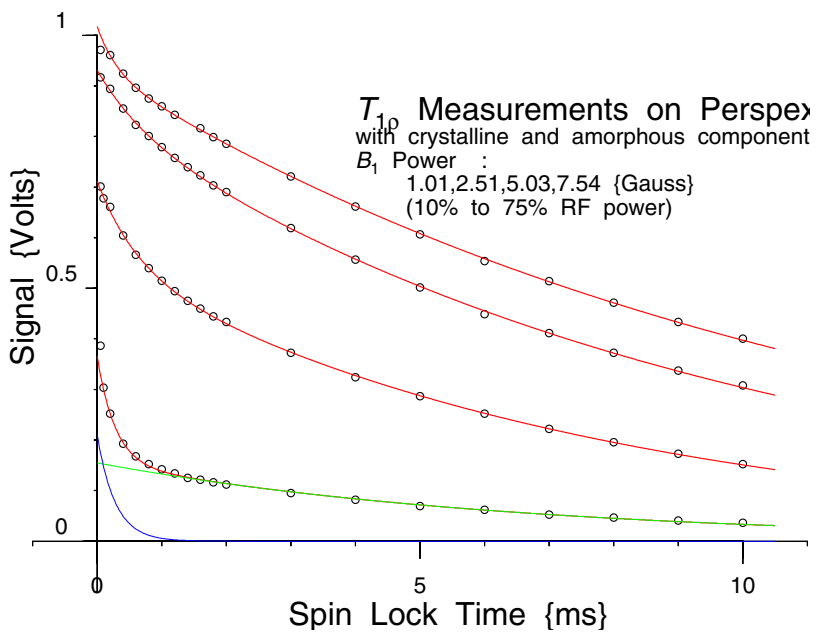

Fig. 11 Showing 2 component $\mathrm{T}_{1} \rho$ decays at lower spin-lock powers, measured on an Acrylic polymer at room temperature, using a set of spin-locking measurements with RF powers from 1 to 7.5 Gauss. Component ratios are affected by the crystalline/amorphous ratio and the ability of the $B_{1}$ pulse to maintain spin-locking in each component. These suggest that the local-field fluctuations in the crystalline component are a bit under 0.6 Gauss

decay shape is closer to a Gaussian, rather than exponential, due to dipolar line-splitting, as shown by the built-in fitting in the lab-Tools spectrometer.

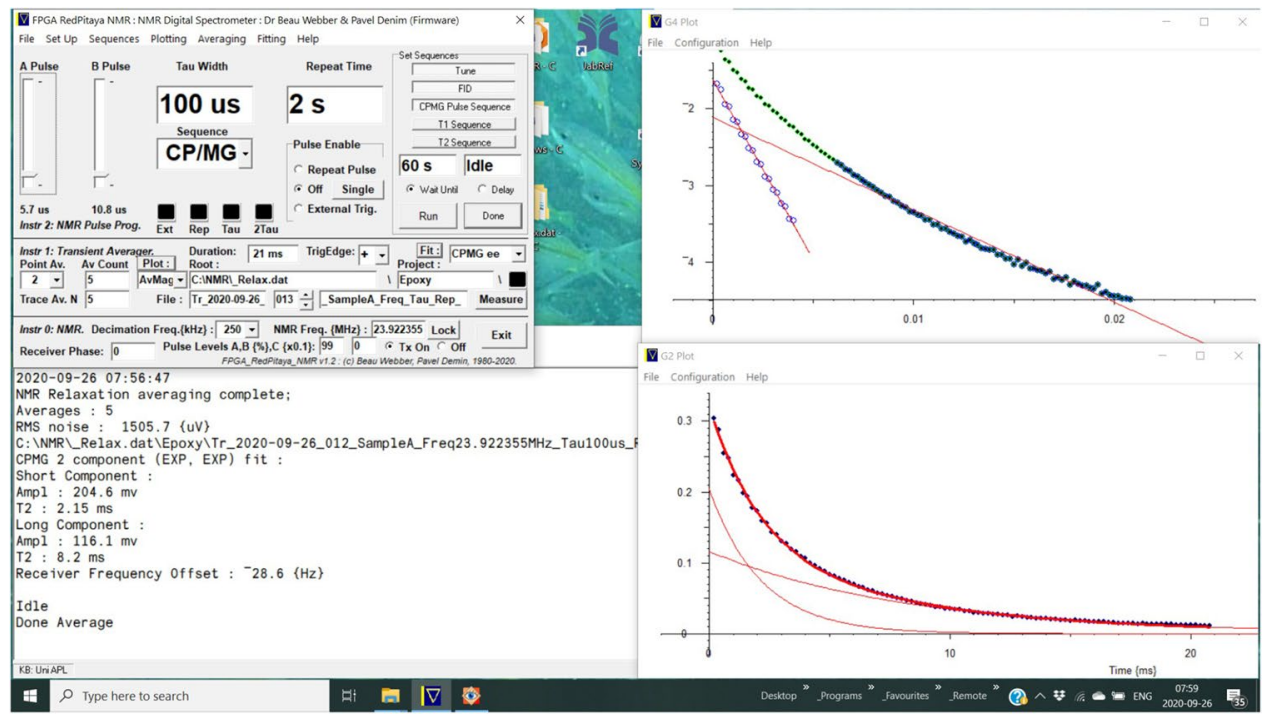

Fig. 10 Showing a 2 component T2 decay, measured on a white rubber sample using a Carr-Purcell-Meibom-Gill (CPMG) sequence. The parameters for the CPMG measurement are set in the graphical user interface (top left), with point sampling at 100 $\mu \mathrm{s}$ for $21 \mathrm{~ms}$, and measuring every $2 \mathrm{~s}$ to allow for $\mathrm{T}_{1}$ recovery. A double-exponential fit is requested, and after 5 averages first a log- lin fit is performed (top right). Two straight-line fits extract initial parameters for a subsequent full non-linear double-exponential fit (bottom right). The fitted parameters are then shown (bottom left) and saved to file. Decay component amplitude ratios are affected by the crystalline/amorphous ratio in the polymer 
a
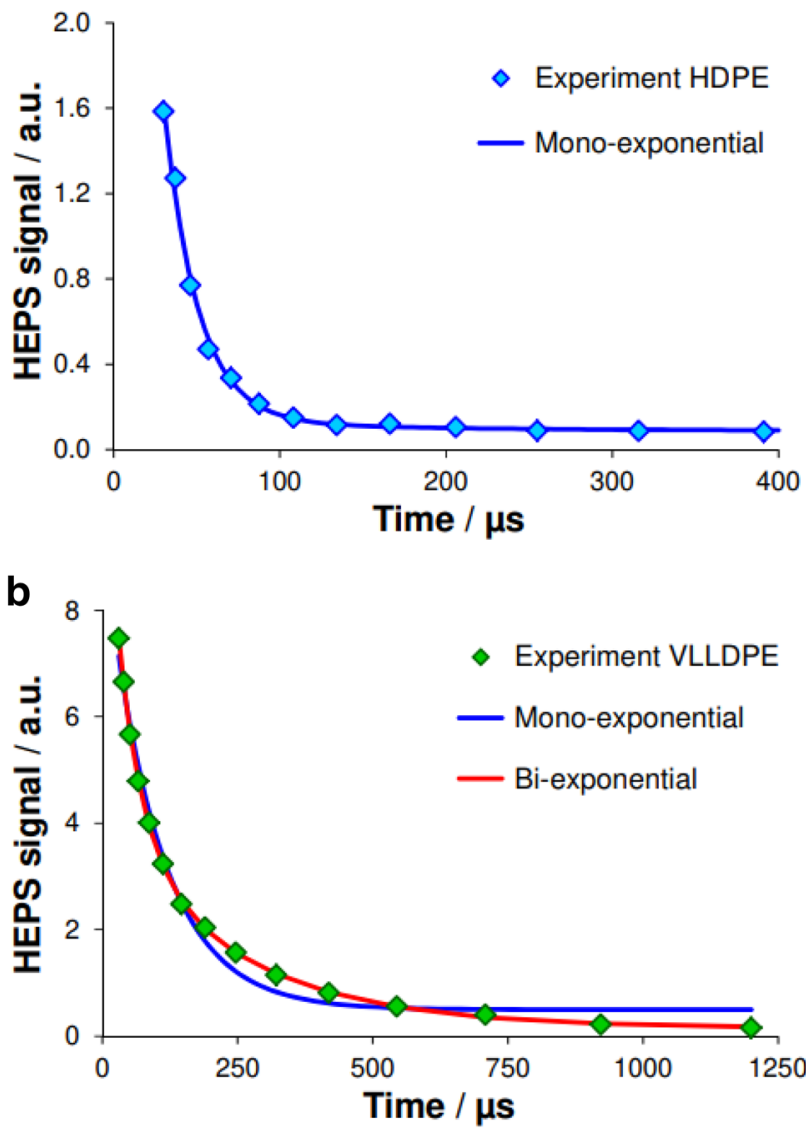

Fig. 12 Mono- and bi-exponential fitting of HEPS data: samples HDPE E (a) and VLLDPE A (b) Spintrack

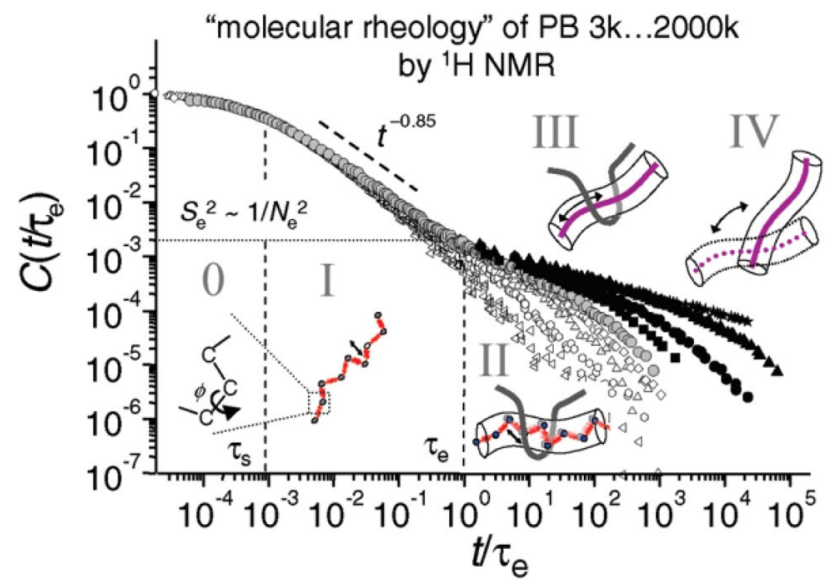

Fig. 13 Entangled polymer dynamics: universal behavior of flexible homopolymers and applicability of the tube model-Bruker Minispec
Hydrocarbon fractions in Barnett Shale

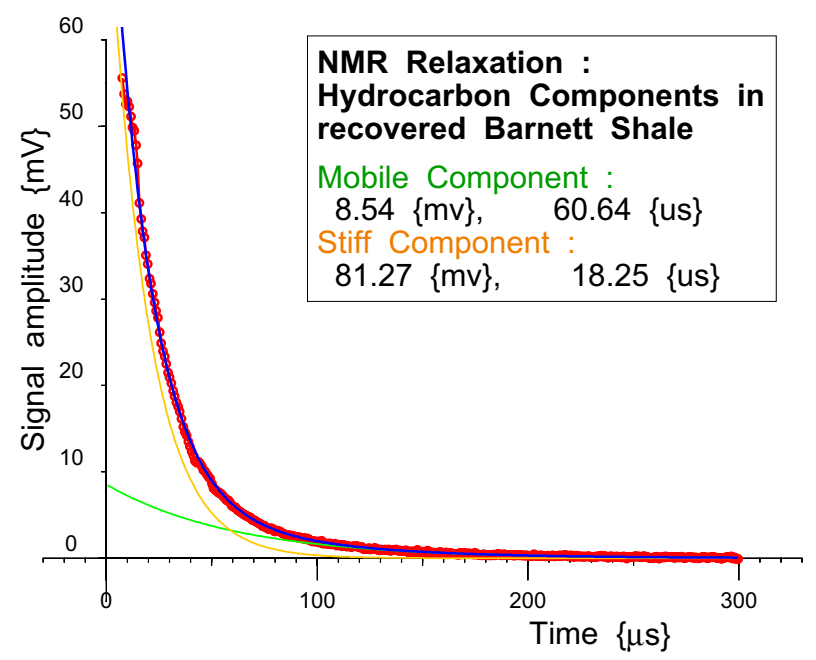

Ln-Lin fitting of hydrocarbons in Barnett Shale

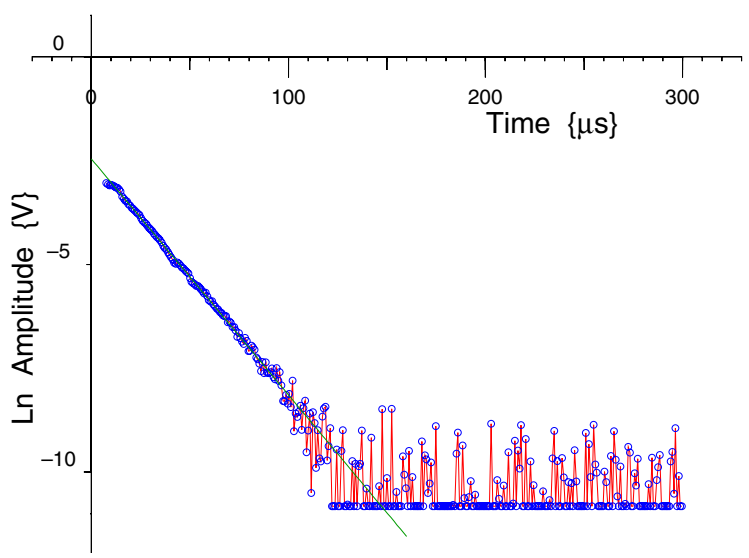

Fig. 14 Again the fitted long-exponential component has been subtracted from the data. The resultant straight line in the logdomain plot clearly demonstrates that the short component is also an exponential

\subsection{Using time-domain NMR to study the properties of polymers}

Polymers : Form a major class of materials which are often studied by time-domain NMR.

Example: Rubber (white). See Fig. 10.

There are a range of important material for which timedomain NMR can measure properties that give much information on the sample. Polymers are a particularly important case, where there is frequently a fast $T_{2}$ decay due to a crystalline component, and a longer, perhaps multicomponent decay, from a more amorphous region, which is often described by a polymer tube "reptation" model. 
NMRR: Biochar FID Fits, HTT $300 \mathrm{C}$ to $700 \mathrm{C}$

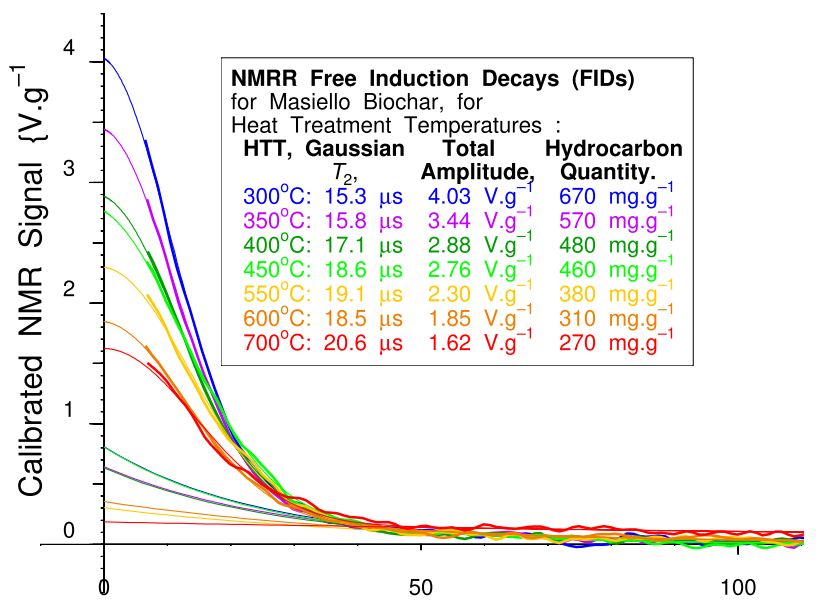

Time $\{\mu \mathrm{s}\}$

Fig. 15 Measurements of hydrocarbon quantity and dynamics/ mobility in the pores of biochar prepared at different heat treatment temperatures (HTTs)

These parameters can also be measured when these polymers or other hydrocarbons are in nano- to micropores, as well as in the bulk materials, see for instance [8], on Polymer chain dynamics under nanoscopic confinements, and [23] for confinement in rock pores and biochar carbon pores.

Figure 10 shows an exponential-exponential $\mathrm{T}_{2}$ decay in rubber, as measured by a Carr-Purcell-Meibom-Gill (CPMG) sequence, using the Lab-Tools built in NMR double-exponential relaxation fit.

In the top right log-log plot the fitted long-exponential component has been subtracted from the data. The resultant straight line clearly demonstrates that the short

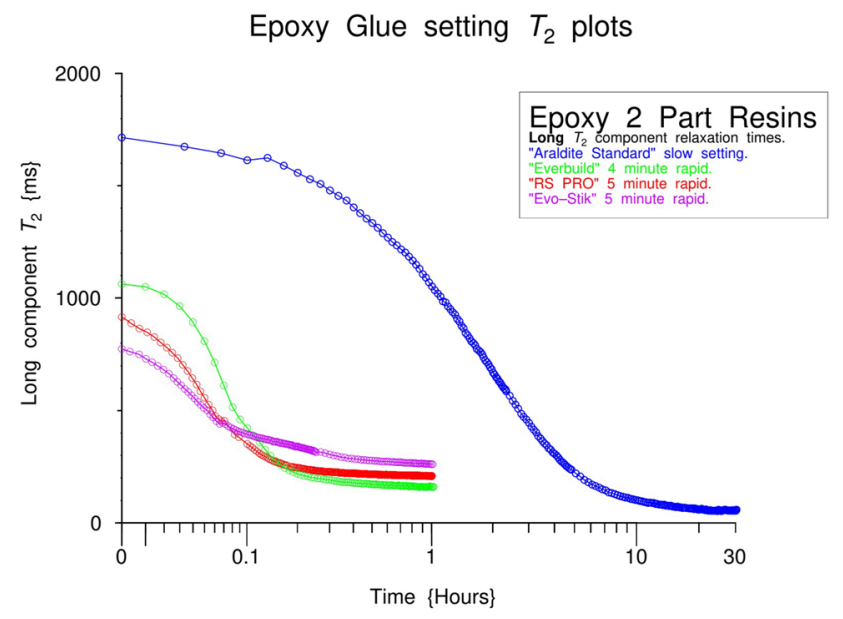

Fig. 16 Molecular mobility changes in reacting Epoxy Resins, monitored by time-domain NMR
Normalised Diffusion graphs vs. Time ${ }^{3}$

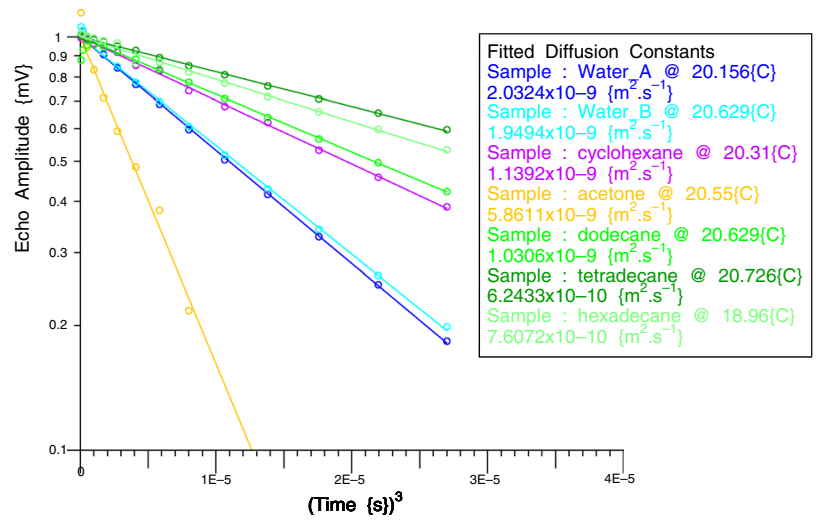

Fig. 17 Normalised liquid self-diffusion vs time ${ }^{3}$, for six mobile liquids

component is also an exponential. These components are affected by the crystalline / amorphous ratio, and this ratio commonly changes with polymer temperature, so is useful for process monitoring and control.

$\mathrm{T}_{1} \rho$ is a particularly powerful method of probing these crystalline / amorphous ratios in polymers, see Fig. 11 for the case of an acrylic polymer, where the normal frequency domain lineshape is a combined broad line(crystalline), giving a very fast decay and a narrower line (amorphous), giving a slower decay. Measuring the $\mathrm{T}_{1} \rho$ decays at $\mathrm{B}_{1}$ fields from about $0.1 \mathrm{mT}$ to $1 \mathrm{mT}$ probes
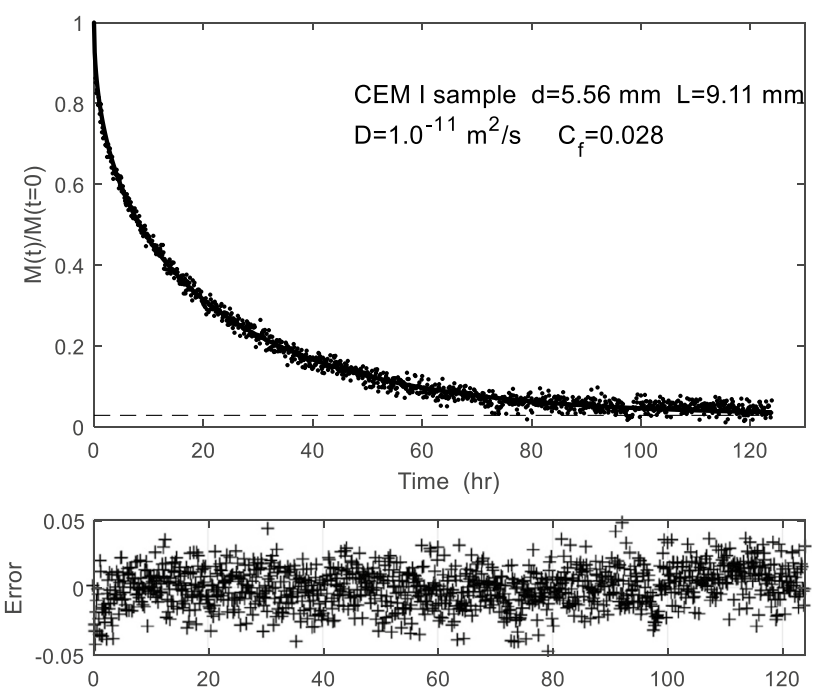

Fig. 18 Diffusion curve on a Portland cement paste (CEM I type). The magnetization amplitude is obtained from FID recordings with a short repeat delay between scans to not polarize long components. The dashed line indicates the fitted asymptotic value $M(t=$ $\infty) / M(t=0)$. The lower panel shows the error between the model and the data. The $T_{2}$ relaxation time distribution of this sample is centered around $0.1 \mathrm{ms-Oxford}$ Maran 


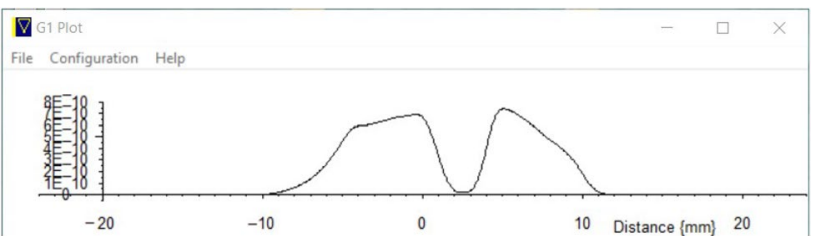

Fig. 19 A 1 dimensional projection image of a structured rubber sample in a linear gradient

motions in the $10 \mathrm{kHz}$ to $100 \mathrm{kHz}$ region, and gives a 2 component decay at lower $B_{1}$, where the crystalline fraction is not fully spin-locked.

Low-field $1 \mathrm{H}$ NMR experiments have been performed on polyethylene using a Spin Track apparatus (Resonance Systems Ltd.) operating at $25.4 \mathrm{MHz}$, to study the crystalline (rigid) and an amorphous (mobile) phases [3]. We show Fig. 5 in the reference as Fig. 12.

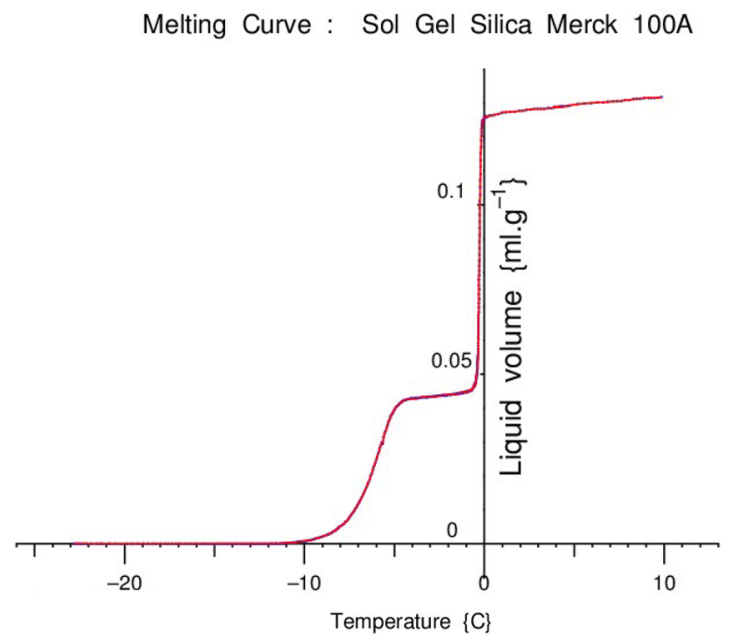

ws0100m_08w: Sol Gel Silica Merck 100A | 2Tau=2ms, Log WR=0.05 0.002 C.min-1 । , 2019-11-11-

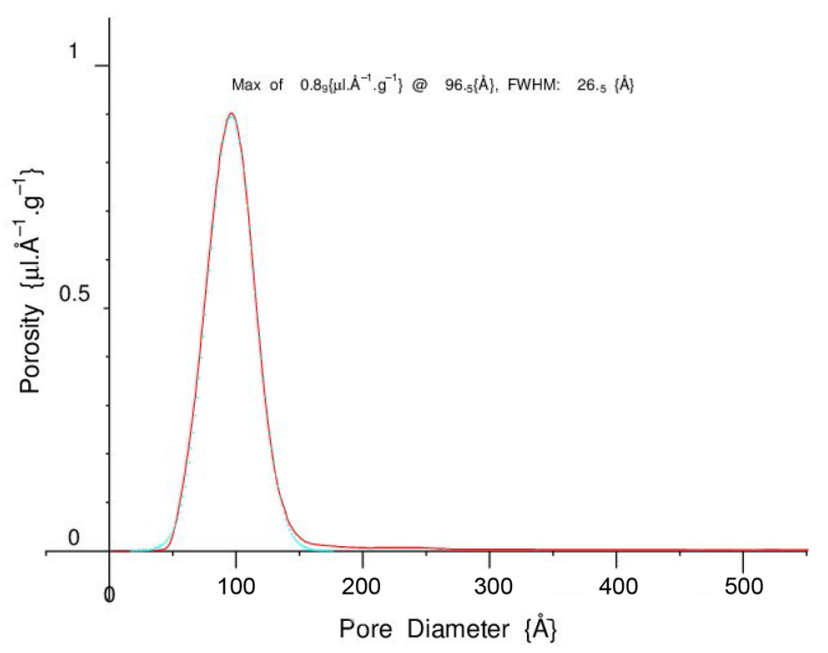

Fig. 20 a) Melting point curve. b) NMRC pore-size distribution, for a nominal $100 \AA$ pore diameter porous silica
Proton multi-quantum NMR experiments have been performed on polymers as a function of temperature, using a Bruker Minispec mq20 $\left(\mathrm{B}_{0}=0.47 \mathrm{~T}\right)$ : time-domain NMR observation of entangled polymer dynamics: universal behavior of flexible homopolymers and applicability of the tube model [4]. The sample temperature was variable over a temperature range between $230 \mathrm{~K}$ and 400 K. Figure 13.

\subsection{Using time-domain NMR to study the properties of hydrocarbons in nano-pores}

We first examining specifically the case of hydrocarbon nanoscopic confinement in rock pores recovered from reservoir wells, in Fig. 14, where we again see a two exponential component decay, the shorter decay being from a less mobile probably tarry component.

Similar measurements have previously been performed on bituminous/oil components in rock pores, see [23], which also discusses measurements on plant resins in biochar porous carbon fired at a range of temperatures, see Fig. 15.

Measurements of hydrocarbon quantity and dynamics / mobility in the pores of biochar prepared at different heat treatment temperatures (HTTs) are shown in Fig. 15 for NMRR free-induction decays for seven biochars, with HTT : $300^{\circ} \mathrm{C}, 350^{\circ} \mathrm{C}, 400^{\circ} \mathrm{C}, 450^{\circ} \mathrm{C}, 550^{\circ} \mathrm{C}, 600^{\circ} \mathrm{C}, 700^{\circ} \mathrm{C}$. [23]. The samples show decreasing amplitude, in order, as increasing amounts of the labile component are driven off with higher HTT. Two component decays are again measured, however the shorter component is now closely fitted by Gaussians, rather than exponentials, due to dipolar line-splitting. This component will be of lower mobility, and is probably attached to the pore wall.

\subsection{NMRR used to monitor chemical processes that result in physical changes to the molecular mobility in the sample}

We studied examples based on a set of different reacting 2-part Epoxy resins, and monitored the changes in the mobility of the resin as setting proceeds. These reaction rates are dependent on resin temperature, which makes time-domain NMR very applicable for process monitoring.

These samples all exhibit a double-exponential magnetization decay. The NMR signal decays were measured by both monitoring the short component $\mathrm{T}_{2}$ of the FID and the also the longer component using a CPMG sequence (see Sect. 2.9), monitoring for over a day in the slowest reacting case.

"Araldite : Standard

"EverBuild : 4 minute" 
Sol-Gel, MCM-41 + SBA-15 melting-point depressions

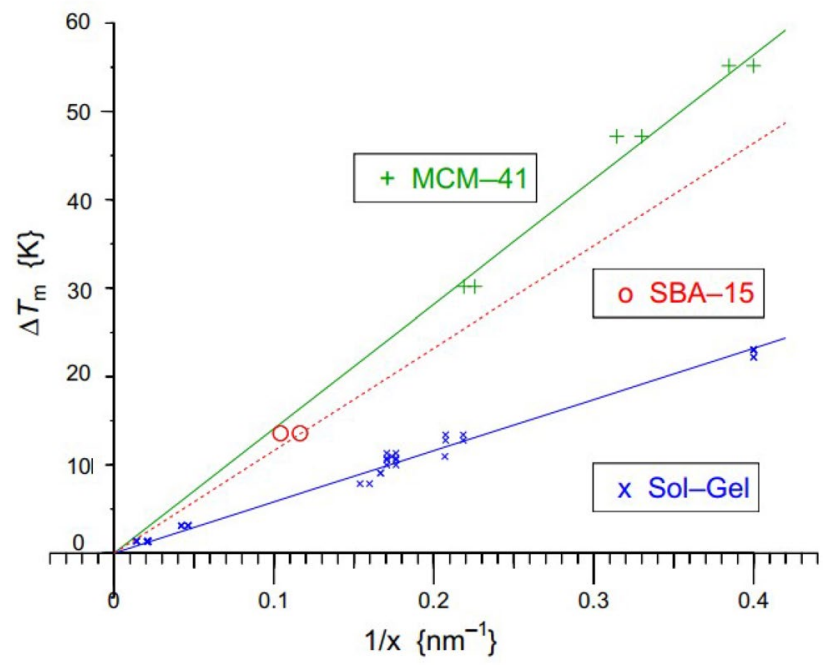

Fig. 21 The relation of melting point depression $\Delta T$ m to inverse pore diameter $\mathrm{x}$, for water in various sol-gel silicas as calibrated by gas adsorption measurements $(\mathrm{x})$, compared with melting point depressions for water in templated cylindrical pores: MCM-41 (+) and SBA-15 (F5) (o). The dotted line has twice the slope as the fit to the sol-gel silica data

\section{"RS PRO : Quick Set"}

\section{"Evo-Stik: Quick Set"}

In Fig. 16 we plot the $\mathrm{T}_{2}$ decay times of the longer components on a single-quadrant version of a bi-symmetric log plot-a variant of a log plot that can go to zero or even negative values on one or both axies [21]. This then provides a measure of the degree to which the chemical

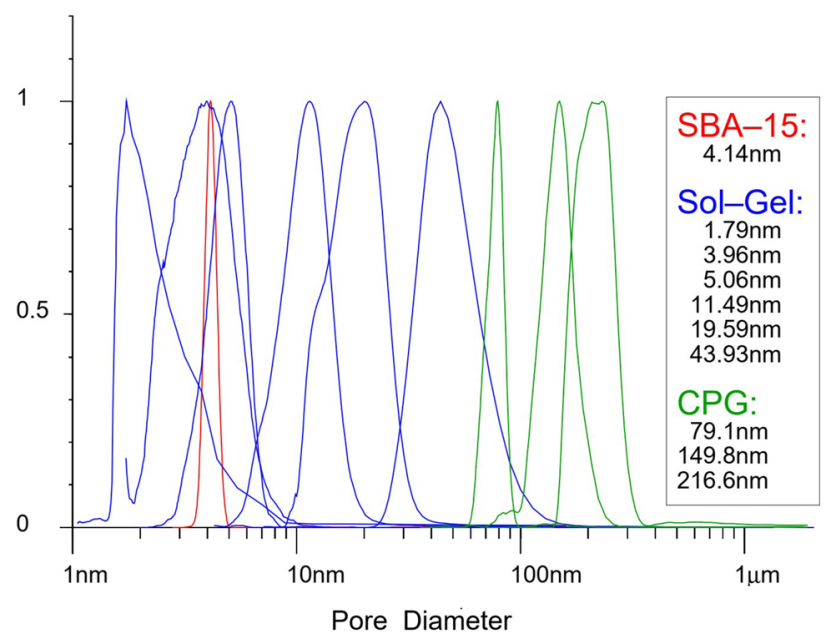

Fig. 22 Normalised pore-size distributions, measured using NMR cryoporometry, using the established kGT value, for a range of porous sol-gel silicas, controlled-pore glasses, and a templated SBA-15 silica

\section{SN Applied Sciences}

rock_2019: NMR-C Pore Size Distributions

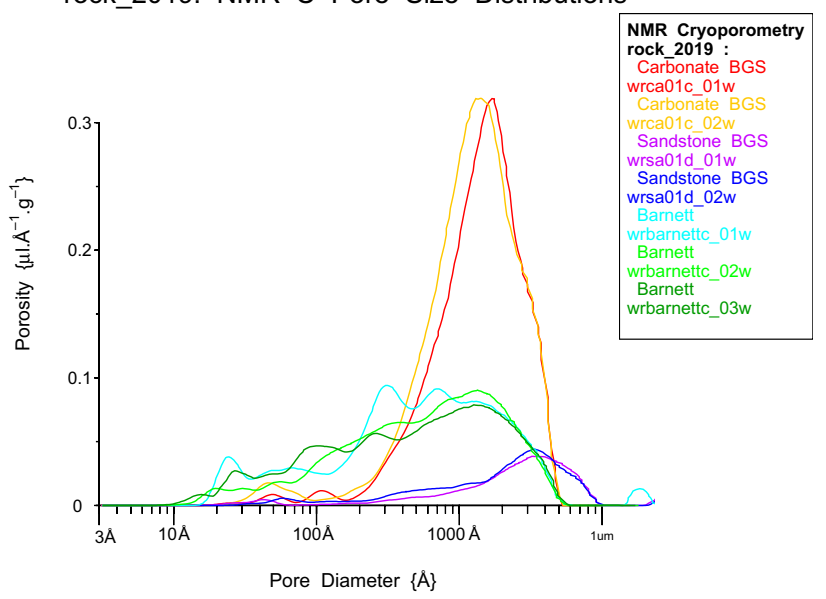

Fig. 23 Repeated NMR cryoporometry pore-size distribution measurements on 3 porous rocks, a Carbonate, a Barnett Shale and a Sandstone

reactions have proceeded, and of the rigidity of the resultant resin.

\subsection{Program requirements for process control}

A number of particular features have been found to be necessary when making extended series of measurements for process control. A particularly important feature is the ability to repeatedly interleave various standard relaxation measurements.

It is also not always required that one continually measures as fast as possible, for process monitoring, it is more important to have set times so as to measure at a regular interval. This both helps limit the amount of data collected, for measurement sequences that may last weeks or months, but also provided suitable spacing for the plotting of the data without having to re-bin the measured data.

An example of the use of time-domain NMR for process control, is the use of time-domain NMR spectrometers to measure online in the factory environment: wood veneer, wood pulp fiber and sludge.

\subsection{NMR used to measure the self-diffusion constants in liquids}

A very significant tool for material science, and process monitoring and control is accessing liquid self-diffusion constants using time-domain NMR, see Fig. 17. 


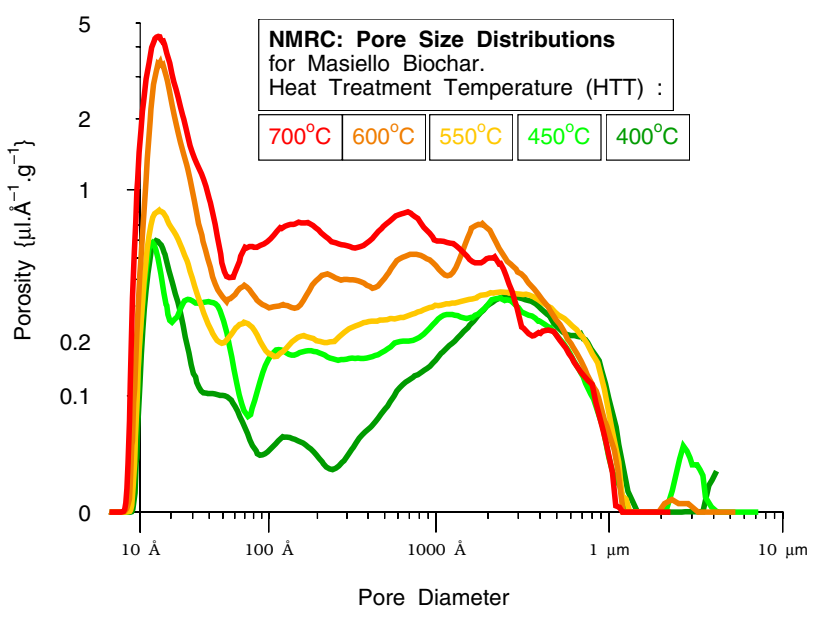

Fig. 24 NMR cryoporometric measurements on five biochars, with different heat treatment temperatures (HTTs)

Here we are measuring the self- diffusion constants for a number of different liquids, by monitoring the NMR echo amplitude in a constant linear magnetic gradient at a number of time $(\tau)$ intervals. We then plot the normalised liquid self-diffusion vs time ${ }^{3}$, please see the original work by Torrey : Bloch equations with diffusion terms : [16]. Self-diffusion constants are highly dependent on liquid temperature, which also makes them very applicable for process monitoring.

\subsection{Measuring diffusion in difficult materials}

"Cement based material are difficult porous media to characterize and there is no exception concerning the

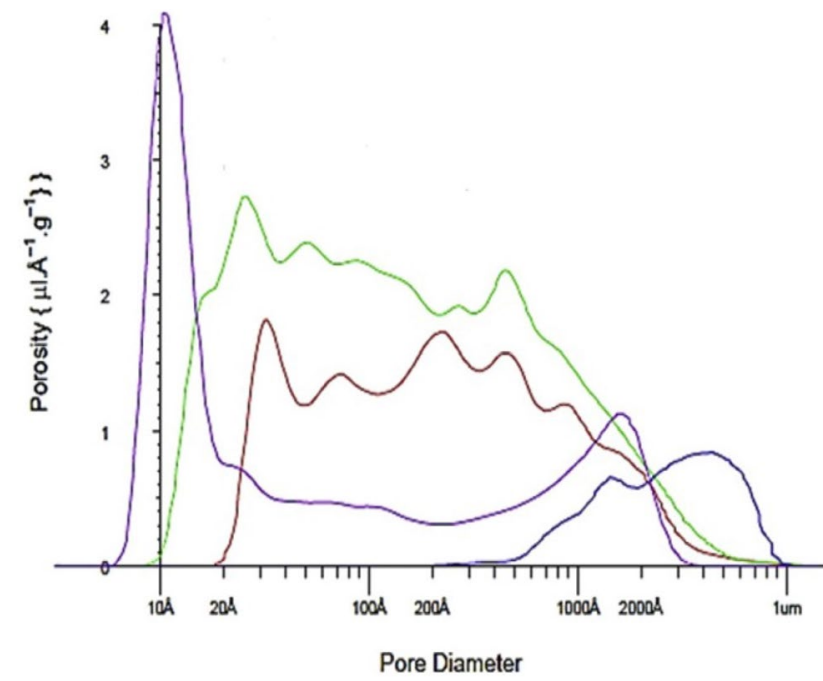

Fig. 25 NMR cryoporometric measurements on four biochars, with different preparations
NMR technique. A deuterium diffusion tracer approach to measure diffusion coefficient in the case of very short NMR relaxation times, too short for NMR pulsed field gradient sequences ( $T_{1}$ or $T_{2}$ below $1 \mathrm{~ms}$ )." Fig. 18 is Fig. 3 from Diffusion of water in industrial cement and concrete [6], and was measured using an Oxford Instruments MQR / Maran time-domain NMR spectrometer with probe size varying from 10 up to $30 \mathrm{~mm}$.

\subsection{Using time-domain NMR spectrometers to make MRI images}

The case of using a static 1D gradient in the main magnet $B_{0}$ field : An image of a structured rubber sample. By placing the sample in a magnetic gradient in the $X$ direction, the different magnetic fields in the sample can be spread into a 1 dimensional projection, by a simple Fourier Transform, see Fig. 19. By taking the magnitude of this Fourier Transform we obtain a 1D projection of the sample density. MRI and NMRR protocols may be combined to produce functional images.

\section{NMR cryoprometry for measuring nano- to micro- pore size distributions}

We have already introduced the NMR Cryoporometrytechnique :

NMRC is a technique where the protocol is that one adds a liquid to the pores, freezes the liquid, and then uses NMRR as a robust method to measure the liquid melted as the sample is warmed.

For water as the probe liquid in templated porous silica of nominal $100 \AA$ pore diameter, see Fig. 20a. Firstly we see the measured melting curve, with a first step, as the sample is warmed, at about $-6 \mathrm{C}$, where the liquid in the pore melts. There is then a plateau, with a further melting point at about the bulk melting point for water at $\mathrm{OC}$, as the liquid around the silica grains melts.

The melting point depression from the usual bulk melting point is inversely proportional to pore diameter. Thus in Fig. 20b, we see that the melting event in the pores has been transformed into a pore size distribution curve, see Sect. 4.

We have extended the NMRC technique for measuring distributions of pore sizes over 3 orders of magnitude in pore dimensions. 
Fig. $261 \mathrm{D}$ spatially resolved pore sizes-3 different sol-gel silicas separated by Teflon spacers, in a $5 \mathrm{~mm}$ diameter NMR tube

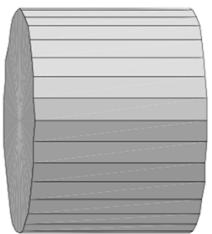

$500 \AA$ Silica

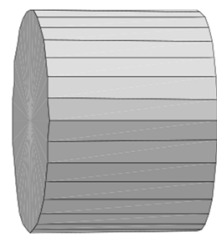

$140 \AA$ Silica

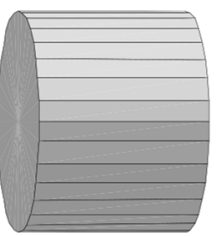

$60 \AA$ Silica

(a) $1 D$ porous silica phantom with axial structure in the $y$ direction.

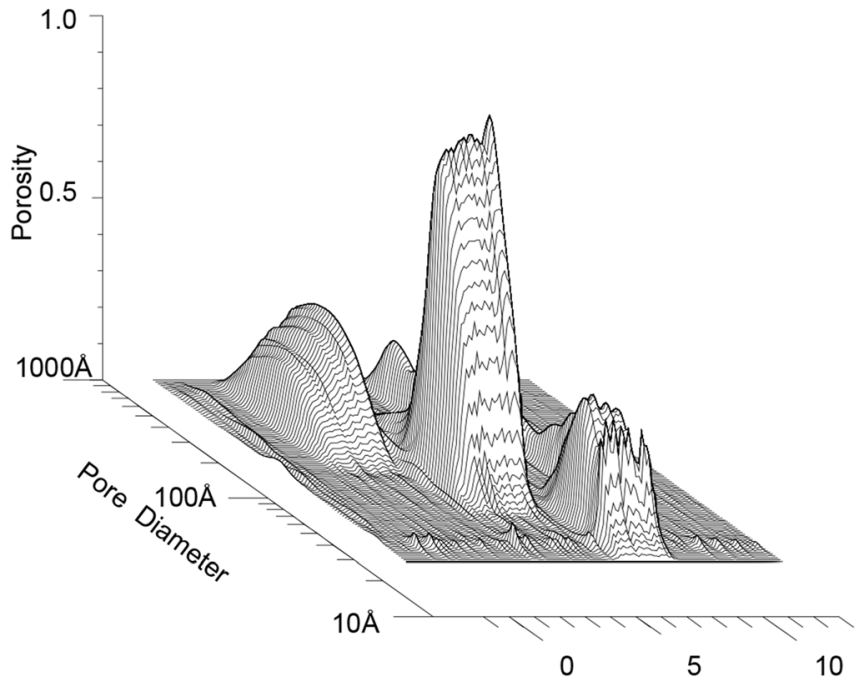

Axial Position $\{\mathrm{mm}\}$

(b) $1 D$ resolved porosity for 3 part phantom.

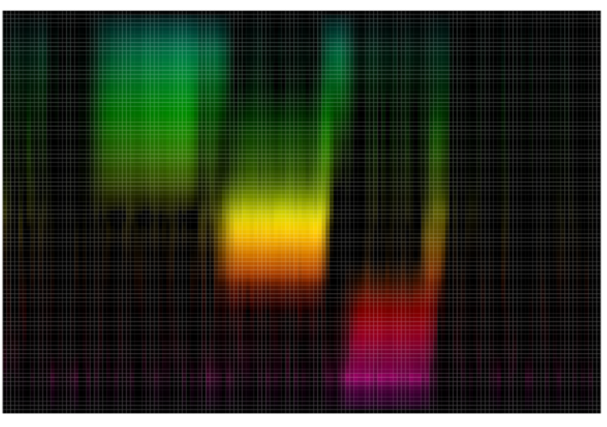

(c) Colour map of Pore Size.

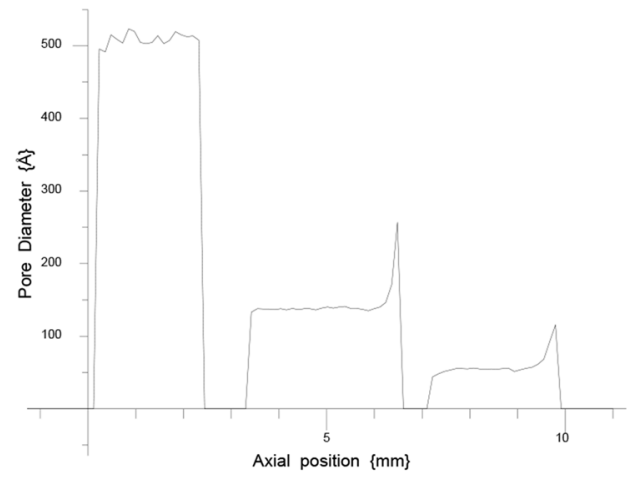

(d) Median pore size as a function of axial position, for the phantom constructed from nominal $500 \AA, 140 \AA$ and $60 \AA$ silicas. 


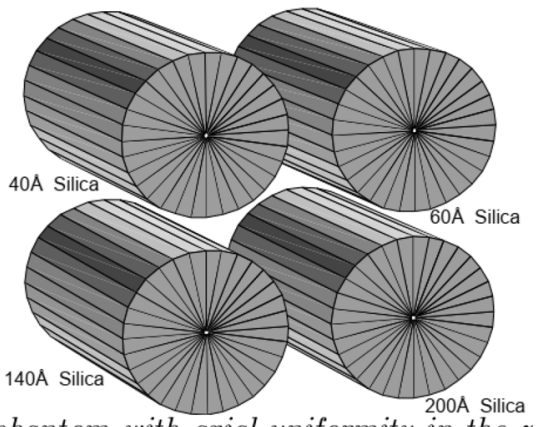

$2 D$ silica phantom with axial uniformity in the $z$ direction.

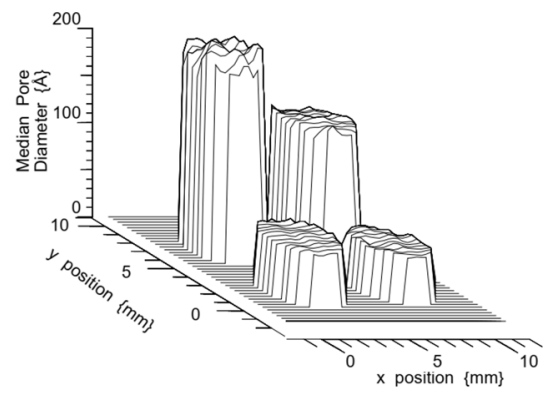

A map of the Median Pore Size for the four tube phantom, rotated for ease of viewing.

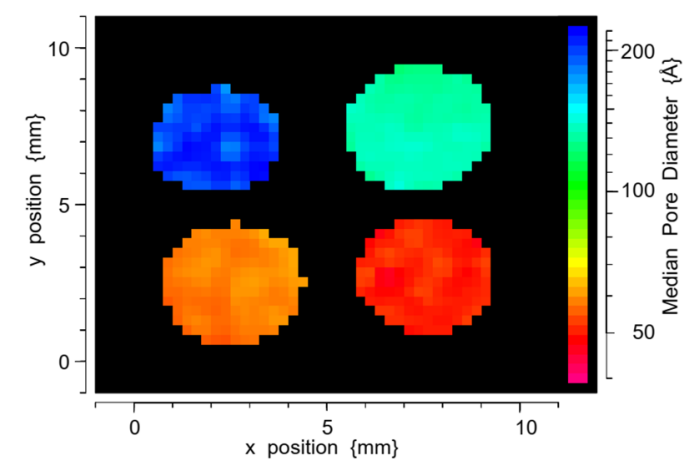

$L_{10}$ Median Pore Diameter mapped to Hue, for the four tube phantom. Blue : $200 \AA$, Cyan : $140 \AA$, Orange : $60 \AA$, Red : $40 \AA$.

Fig. $272 \mathrm{D}$ spatially resolved pore sizes -4 different sol-gel silicas in a set of $5 \mathrm{~mm}$ diameter NMR tubes

The NMRC protocol can be combined with the MRI protocol, to produce 1D, 2D or 3D resolved porosity [13-15], see Sect. 4.3 .

\section{NMR cryoporometry (NMRC) theory}

Josiah Willard Gibbs and three different Thomsons (James Thomson, William Thomson (later Lord Kelvin) and J.J. Thomson) applied experiment, thermodynamics and generalised dynamics to produce an equation that well describes the phase-change behaviour of liquids in confined geometry; the Gibbs-Thomson equation for the melting point depression, $\Delta T m$, for a small isolated spherical crystal, of diameter $x$, in its own liquid, may be expressed as [10]:

$\Delta T_{m}=T_{m}^{\infty}-T_{m}(x)=\frac{4 \sigma_{c l} T_{m}^{\infty}}{x \Delta H_{f} \rho_{s}}$

A development of the Gibbs-Thomson equation has been discussed that relates these phase changes so that the pore area $a_{p}$ and volume $v_{p}$ are related to the melting point depression $[18,20,25]$ :

$\Delta T_{m}=T_{m}-T_{m}(x) \approx \frac{a_{p}}{v_{p}} \cdot \frac{\sigma_{s l} T_{m} \cos (\varphi)}{\Delta H_{f} \rho_{s}} \approx \frac{k_{d} \sigma_{s} T_{m}}{x \Delta H_{f} \rho_{s}}$

For many purposes this may be simplified so that the pore diameter $\mathrm{x}$ is related to a melting point depression $\{\mathrm{K}\}=\Delta$ $\mathrm{T}_{m}=\mathrm{k}_{G T} / \mathrm{x}$ where we are grouping all the thermodynamic terms into a single constant, $\mathrm{k}_{G T}\left\{\mathrm{~K} . \AA \AA^{\mathrm{A}}\right\}$-the Gibbs-Thomson coefficient-usually established by experiment [19, 20], see Fig. 21, and then applied in Fig. 22.

\subsection{NMRC measurement of poresize distributions in 3 porous rock types}

With this Peltier cooled variable-temperature probe we have very good thermal control, and a very uniform sample temperature, which gives us an upper pore-size resolution which now extends to over $1 \mu \mathrm{m}$. By measuring each rock twice we obtain a good estimate of the repeatability of the NMRC measurements, Fig. 23.

\subsection{NMR cryoporometric measurements on biochars, with different preparation methods}

4.2.1 NMR cryoporometric measurements on five biochars, with different heat treatment temperatures (HTTs)

An NMR study of porous rock and biochar containing organic material, for biochar prepared at five different HTTs: $400^{\circ} \mathrm{C}, 450^{\circ} \mathrm{C}, 550^{\circ} \mathrm{C}, 600^{\circ} \mathrm{C}, 700^{\circ} \mathrm{C}$. The samples show increasing pore volumes, in order, as increasing amounts of the labile component are driven off with higher HTT, see Fig. 24 [23]. 
Fig. $283 \mathrm{D}$ spatially resolved pore sizes-A structured sample prepared from 2 different sol-gel silicas, in a $10 \mathrm{~mm}$ diameter test tube

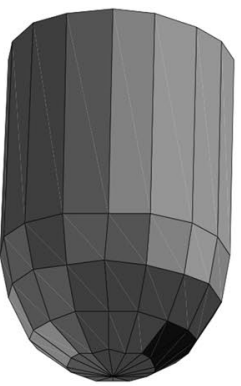

All Silica

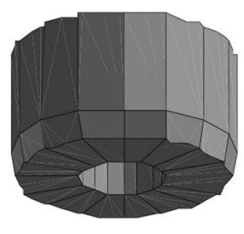

$500 \AA$ Silica

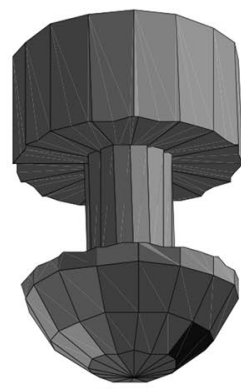

$60 \AA$ Silica

(a) Idealised $3 D$ Structure of $500 \AA$ plus $60 \AA$ silica phantom.

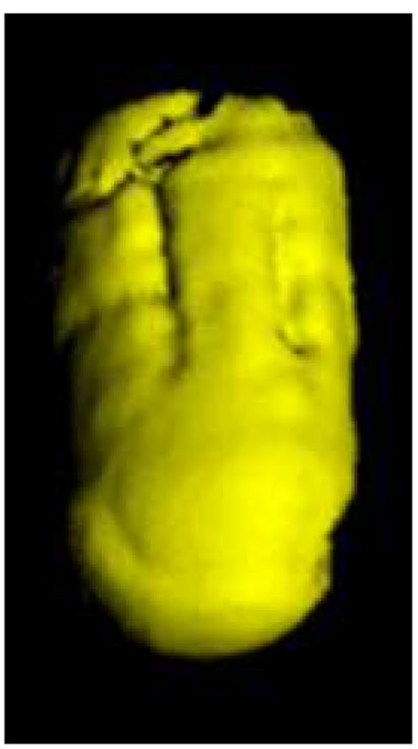

Undifferentiated Silica

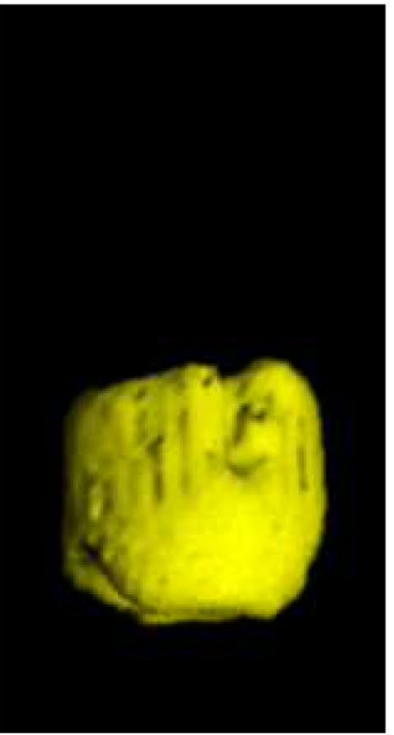

$500 \AA$ Silica

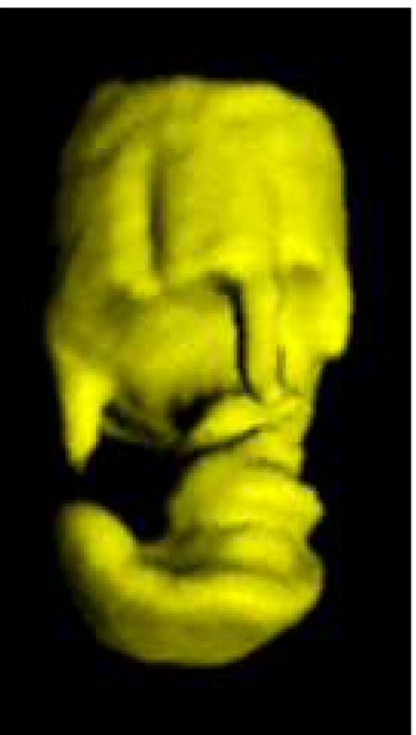

$60 \AA$ Silica

(b) $3 D$ resolved Pore Size Structure.

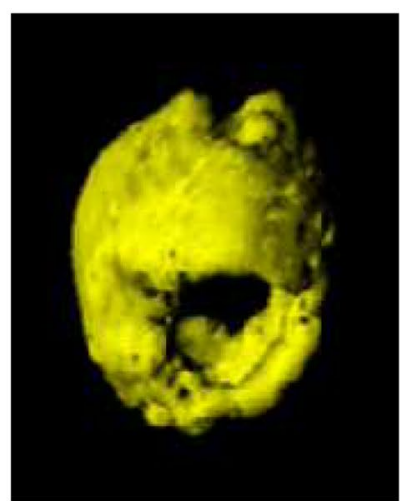

(c) Underneath view of $500 \AA$ Silica. 


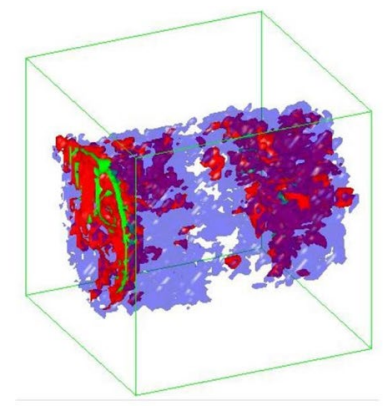

$3 \mathrm{D}$ rock core image $\mathrm{T}=268 \mathrm{~K}$

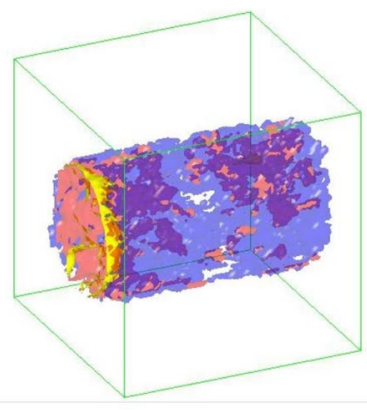

$3 \mathrm{D}$ rock core image $\mathrm{T}=295 \mathrm{~K}$ (Chalk reference visible on left)
Fig. $29 \mathrm{MRI}$ of pore size distributions in fault sealing oil reservoir rock core samples by cryoporometric filtering techniques. Mallett $\mathrm{MJD}^{a}$, Clennell $\mathrm{MB}^{b}$, Strange $\mathrm{JH}^{a}$, Fisher $\mathrm{Q}^{c}$ : a School of Physical Sciences, University of Kent, UK; b Centro de Pesquisa em Geofisica e Geologia-IGEO, Universidade Federal da Bahiat Brasil; c Department of Earth Sciences, University of Leeds, UK

\subsubsection{NMR cryoporometric measurements on four biochars, with different preparations, including flash variants}

The characterisation of biochar porosity by NMRC, with a study of its ammonium ion adsorption properties, see Fig. 25 [26]. The lower end of the pore-size resolution now extends to below $1 \mathrm{~nm}$, the upper end to $1 \mu \mathrm{m}$.

\subsection{Combining NMRC and MRI protocols: 1D, 2D and 3D spatially resolved pore sizes}

It is not easy to represent complete pore-size distributions for all of the 1D, 2D and 3D spatially resolved pore size cases. For the 1D case it can be done, see Fig. 26. In the $2 \mathrm{D}$ and $3 \mathrm{D}$ cases progressively less is presentable, see Figs. 27, 28 [13-15, 19]. The 2D and 3D cases needed a NMR spectrometer with a wide-bore magnet.

Figure 29 shows a combined NMRC and MRI protocol image of a porous rock. Thus this is basically distinguishing the locations of large vs small pores in recovered rock sample by using the Gibbs-Thomson effect to selectively freeze the water in the larger pores.

\section{Declarations}

Conflict of interest The author declares that they have no conflict of interest, beyond being a material-scientist who also designs instrumentation, including the Mk1, Mk2 and Mk3 NMR spectrometers, and who has further developed NMR Cryoporometry.

Open Access This article is licensed under a Creative Commons Attribution 4.0 International License, which permits use, sharing, adaptation, distribution and reproduction in any medium or format, as long as you give appropriate credit to the original author(s) and the source, provide a link to the Creative Commons licence, and indicate if changes were made. The images or other third party material in this article are included in the article's Creative Commons licence, unless indicated otherwise in a credit line to the material. If material is not included in the article's Creative Commons licence and your intended use is not permitted by statutory regulation or exceeds the permitted use, you will need to obtain permission directly from the copyright holder. To view a copy of this licence, visit http://creativecommons. org/licenses/by/4.0/.

\section{References}

1. Abragam A (1961) The Principles of nuclear magnetism. Clarendon Press. https://books.google.co.uk/books/about/The_Princ iples_of_Nuclear_Magnetism.html?id=9M8U_JK7K54C

2. Brownstein K, Tarr C (1977) Spin-lattice relaxation in a system governed by diffusion. J Magn Reson 26:17-24

3. Chmelař J, Pokorný R, Schneider P, Smolná K, Bělský P, Kosek J (2015) Free and constrained amorphous phases in polyethylene: interpretation of $1 \mathrm{~h} \mathrm{nmr}$ and saxs data over a broad range of crystallinity. Polymer 58:189-198. https://doi.org/10.1016/j. polymer.2014.12.036. https://www.sciencedirect.com/science/ article/pii/S0032386114011367

4. Chávez FV, Saalwächter K (2011) Time-domain nmr observation of entangled polymer dynamics: universal behavior of flexible homopolymers and applicability of the tube model. Macromolecules. https://doi.org/10.1021/ma1025708

5. Eidmann G, Savelsberg R, Blümler P, Bluümich B (1996) The nmr mouse, a mobile universal surface explorer. J Magn Reson Ser A, article no. 0185(122):104-109. https://ep5.physik.uni-wuerz burg.de/people/pkvogel/2019_zumLesen/01/Eidmann\%20et\% 20al\%20-\%20the\%20NMR\%20MOUSE,\%20a\%20Mobile\%20Uni versal\%20Surface\%20Explorer\%20-\%201996.pdf

6. Fleury M, Berthe G, Chevalier T (2019) Diffusion of water in industrial cement and concrete. Magn Reson Imaging 56:3236. doi: https://doi.org/10.1016/j.mri.2018.09.010. https://www. sciencedirect.com/science/article/pii/S0730725X1830328X. Porous Media

7. Halbach K (1980) Design of permanent multipole magnets with oriented rare earth cobalt material. Nucl Inst Methods 169(1):110. https://doi.org/10.1016/0029-554X(80)90094-4

8. Kimmich R, Fatkullin N, Mattea C, Fischer E (2005) Polymer chain dynamics under nanoscopic confinements. MAGNETIC RESONANCE IMAGING 23(2, Sp. Iss. SI):191-196. doi: https://doi.org/ 10.1016/j.mri.2004.11.050. 7th International Conference on Magnetic Resonance in Porous Media (MRPM7), Palaiseau, FRANCE, JUL 04-08, 2004

9. Mitchell J, Stark S, Strange J (2005) Probing surface interactions by combining NMR cryoporometry and NMR relaxometry. J Phys D Appl Phys 38(12):1950-1958. https://doi.org/10.1088/00223727/38/12/015

10. Mitchell J, Webber J, Strange J (2008) Nuclear magnetic resonance cryoporometry. Phys Rep 461(1):1-36

11. Norris MO, Strange JH (1969) A nuclear magnetic resonance sample temperature controller using liquid nitrogen injection. J Phys E Sci Instrum 2(12):1106-1108

12. Raich $H$, Blümler $P$ (2004) Design and construction of a dipolar halbach array with a homogeneous field from identical bar magnets: NMR mandhalas. Concepts Magn Reson Part B Magn Reson Eng 23B:16-25. https://doi.org/10.1002/cmr.b.20018 
13. Strange J, Webber J (1997) Multidimensionally resolved pore size distributions. Appl Magn Reson 12(2-3):231-245

14. Strange J, Webber J (1997) Spatially resolved pore size distributions by NMR. Meas Sci Technol 8(5):555-561

15. Strange J, Webber J, Schmidt S (1996) Pore size distribution mapping. Magn Reson Imaging 14(7-8):803-805

16. Torrey HC (1956) Bloch equations with diffusion terms. Phys Rev 104(563):

17. Webber B (2012) NMR looks deep inside nooks and crannies. Physics 5:14. https://doi.org/10.1103/Physics.5.14

18. Webber J (2003) A generalisation of the thermoporisimetry Gibbs-Thomson equation for arbitrary pore geometry. Magn Reson Imaging 21(3-4):428. https://doi.org/10.1016/S0730$725 \times(03) 00172-3$

19. Webber JBW (2000) The characterisation of porous media. PhD, Physics, University of Kent at Canterbury, UK. http://www.kent. ac.uk/physical-sciences/publications/theses/jbww.html

20. Webber JBW (2010) Studies of nano-structured liquids in confined geometry and at surfaces. Prog Nucl Mag Res Sp 56:78-93. https://doi.org/10.1016/j.pnmrs.2009.09.001

21. Webber JBW (2013) A bi-symmetric log transformation for widerange data. Meas Sci Technol 24(2):027001. https://doi.org/10. 1088/0957-0233/24/2/027001

22. Webber JBW (2020) Some applications of a field programmable gate array based time-domain spectrometer for NMR relaxation and NMR cryoporometry. Appl Sci 10(8):2714. https://doi.org/ 10.3390/app10082714
23. Webber JBW, Corbett P, Semple KT, Ogbonnaya U, Teel WS, Masiello CA, Fisher QJ, Valenza-II JJ, Song YQ, Hu Q (2013) An NMR study of porous rock and biochar containing organic material. Microporous Mesoporous Mater 178:94-98. In: Proceedings of the 11th International Bologna Conference on Magnetic Resonance in Porous Media (MRPM11), University of Surrey, 2012. https://doi.org/10.1016/j.micromeso.2013.04.004

24. Webber JBW, Demin P (2019) Credit-card sized field and benchtop $\mathrm{nmr}$ relaxometers using field programmable gate arrays. Magn Reson Imaging 56:45-51. https://doi.org/10.1016/j.mri. 2018.09.018. http://www.sciencedirect.com/science/article/pii/ S0730725X18303102

25. Webber JBW, Dore JC, Strange JH, Anderson R, Tohidi B (2007) Plastic ice in confined geometry: the evidence from neutron diffraction and NMR relaxation. J Phys Condens Matter 19:415117. https://doi.org/10.1088/0953-8984/19/41/415117. http://stacks. iop.org/0953-8984/19/415117

26. Wong J, Webber J, Ogbonnaya U (2019) Characteristics of biochar porosity by $\mathrm{nmr}$ and study of ammonium ion adsorption. J Anal Appl Pyrolysis 143:104687. https://doi.org/10.1016/j.jaap. 2019.104687. http://www.sciencedirect.com/science/article/pii/ S0165237019302013

Publisher's Note Springer Nature remains neutral with regard to jurisdictional claims in published maps and institutional affiliations. 